\title{
1 Condensation of Rubisco into a proto-pyrenoid in higher plant chloroplasts
}

3 Nicky Atkinson ${ }^{1}$, Yuwei Mao ${ }^{1}$, Kher Xing Chan ${ }^{2}$, Alistair J. McCormick ${ }^{1}$

$4 \quad{ }^{1}$ SynthSys \& Institute of Molecular Plant Sciences, School of Biological Sciences, King's

5 Buildings, University of Edinburgh, EH9 3BF, UK

$6{ }^{2}$ Carl R. Woese Institute for Genomic Biology, University of Illinois at Urbana-Champaign, 71206 Gregory Drive, Urbana IL 61801.

\section{Summary}

10 Photosynthetic $\mathrm{CO}_{2}$ fixation in plants is limited by the inefficiency of the $\mathrm{CO}_{2}$-assimilating enzyme Rubisco (D-ribulose-1,5-bisphosphate carboxylase/ oxygenase) ${ }^{1-3}$. In plants possessing the C3 pathway, which includes most major staple crops, Rubisco is typically evenly distributed throughout the chloroplast stroma. However, in almost all eukaryotic algae Rubisco aggregates within a microcompartment known as the pyrenoid, in association with a $\mathrm{CO}_{2}$-concentrating mechanism that improves photosynthetic operating efficiency under conditions of low inorganic carbon ${ }^{4}$. Recent work has shown that the pyrenoid matrix is a phase-separated, liquid-like condensate ${ }^{5}$. In the alga Chlamydomonas reinhardtii, condensation is mediated by two components: Rubisco and the linker protein EPYC1 (Essential Pyrenoid Component 1$)^{6,7}$. Here we show that expression of mature EPYC1 and a plant-algal hybrid Rubisco leads to spontaneous condensation of Rubisco into a single phase-separated compartment in Arabidopsis chloroplasts, with liquid-like properties similar to a pyrenoid matrix. The condensate displaces the thylakoid membranes and is enriched in hybrid Rubisco containing the algal Rubisco small subunit required for phase separation. Promisingly, photosynthetic $\mathrm{CO}_{2}$ fixation and growth is not impaired in stable transformants compared to azygous segregants. These observations represent a significant initial step towards enhancing photosynthesis in higher plants by introducing an algal $\mathrm{CO}_{2}$-concentrating mechanism, which is predicted to significantly increase the efficiency of photosynthetic $\mathrm{CO}_{2}$ uptake ${ }^{8,9}$. 


\section{Main Text}

Rubisco catalyses net $\mathrm{CO}_{2}$-fixation in all photosynthetic organisms but also reacts with oxygen, leading to a wasteful loss of previously fixed $\mathrm{CO}_{2}$, nitrogen and energy ${ }^{1}$. In plants with the C3 photosynthetic pathway (e.g. rice, wheat and soybean), Rubisco has a relatively slow turnover rate and operates under sub-optimal $\mathrm{CO}_{2}$ concentrations of less than half that required for maximal rates of carboxylation ${ }^{10-12}$. Improving the operating efficiency of Rubisco remains a key strategy to increase productivity in $\mathrm{C} 3 \mathrm{crops}^{13}$. This is an important goal given the steep trajectory of crop yield improvements required to meet the demands of our rising global population ${ }^{14}$.

Most unicellular eukaryotic photosynthetic organisms and some non-vascular land plants have evolved highly efficient $\mathrm{CO}_{2}$-concentrating mechanisms (CCMs) that condense Rubisco into a micro compartment within the chloroplast called the pyrenoid ${ }^{4,15}$. The CCM functions to enrich the pyrenoid with high concentrations of $\mathrm{CO}_{2}$ to enhance the performance of Rubisco carboxylation and suppress oxygenation ${ }^{16}$. Introduction of such CCMs into C3 plants is predicted to lead to significant increases in $\mathrm{CO}_{2}$-fixation efficiency and biomass yield ${ }^{14,17,18}$. Recent work in the model green alga Chlamydomonas reinhardtii (hereafter Chlamydomonas) has shown that the pyrenoid is a liquid like-condensate that undergoes liquid-liquid phase separation when the CCM is active ${ }^{5}$. Phase separation is facilitated by multivalent interactions between the intrinsically disordered Rubisco-binding protein EPYC1 and the small subunit of Rubisco $(\mathrm{SSU})^{7}$, of which residues on the $\alpha$-helices of the native SSU isoforms are critical for binding to EPYC $1^{19,20}$. Previously we have shown that Arabidopsis can assemble a functional plant-algal hybrid Rubisco complex, with the native large subunit of Rubisco (LSU) and an SSU from Chlamydomonas, which has similar kinetic properties to wild-type (WT) Arabidopsis Rubisco ${ }^{21}$. However, expression of the full coding sequence of EPYC1 in Arabidopsis with plant-algal hybrid Rubisco did not result in Rubisco condensation ${ }^{19}$. Evidence from Chlamydomonas and in vitro studies suggested that an appropriate stoichiometric balance between EPYC1 and Rubisco, and possibly additional regulatory components, might be required for efficient phase separation ${ }^{4,5,7,22}$.

We sought to test whether high levels of expression of a mature form of EPYC1, could lead to phase separation in a higher plant chloroplast. EPYC1 was truncated according to the predicted transit peptide cleavage site between residues $26(\mathrm{~V})$ and $27(\mathrm{~A})^{19}$. A dual GFP expression system was developed to achieve high levels of EPYC1 expression and a favourable stoichiometry with Rubisco. This consisted of a binary vector containing two gene expression cassettes, each encoding mature EPYC1 with an Arabidopsis chloroplastic signal peptide and 
fused to a different version of GFP (turboGFP (tGFP) or enhanced GFP (eGFP)) to avoid possible recombination events (Fig. 1a, Extended Data Fig. 1). The dual GFP construct (EPYC1-dGFP) was transformed into WT plants or the Arabidopsis la3b Rubisco mutant complemented with an SSU from Chlamydomonas $\left(\mathrm{S}_{2} \mathrm{Cr}\right)^{21}$. The resulting transgenic plants (Ep) expressed both EPYC1::eGFP and EPYC1::tGFP, of which the latter was generally more abundant at the protein level (Fig. 1b, Extended Data Fig. 2). Previously, immunoblots against full length EPYC1 expressed in S2 $\mathrm{Cr}$ or WT plants showed additional lower molecular weight bands indicative of proteolytic degradation ${ }^{19}$. Here, degradation products of the mature EPYC1 were relatively reduced when expressed in $\mathrm{S} 2 \mathrm{Cr}$ compared to WT plants.

The fluorescence signal for EPYC1-dGFP in WT plants was distributed evenly throughout the chloroplast (Fig. 1c, Fig. 2a). In contrast, EPYC1-dGFP in the hybrid S2 $2_{\text {rr }}$ plants showed only a single dense chloroplastic signal. Transmission electron microscopy confirmed the presence of a single prominent condensed complex in the chloroplast stroma (Fig. 1d). The condensates were spherical in shape and displaced native chlorophyll autofluorescence (Fig. 1e, f), indicating that the thylakoid membrane matrix was excluded from the condensate. In protoplasts of leaf mesophyll cells, a condensate was visible in each chloroplast (Extended Data Fig. 3a), and the average size of the condensates was related to the expression level of EPYC1-dGFP (Fig. 1b, Extended Data Fig. 3b, c). We observed that the average diameter of the condensates was $1.6 \pm 0.1 \mu \mathrm{m}\left(\mathrm{n}=126 ; 42\right.$ from three individual $\mathrm{S} 2{ }_{\mathrm{Cr}}$ transgenic lines), which was comparable with that of the size range measured for the Chlamydomonas pyrenoid $(1.4 \pm 0.1 \mu \mathrm{m} ; \mathrm{n}=55)^{23}$ (Fig. 1g, Extended Data Fig. 3c). The estimated volume of the condensates was $2.7 \pm 0.2 \mu \mathrm{m}^{3}$ ( $c a .5 \%$ of the chloroplast volume). Variations in condensate volume within individual $\mathrm{S} 2 \mathrm{Cr}$ transgenic Ep lines were not correlated with chloroplast volume, suggesting that regulation of condensate formation and size was largely independent of chloroplast morphology, and likely limited by the influence of the surrounding network stiffness (i.e. the stroma, which is densely packed with thylakoids) on the dynamics of droplet ripening ${ }^{24}$.

Condensates were also observed when EPYC1-dGFP was expressed in the Arabidopsis $1 a 3 b$ Rubisco mutant complemented with a native Arabidopsis SSU modified to contain the two $\alpha$-helices necessary for pyrenoid formation from the Chlamydomonas SSU $\left(1 \mathrm{~A}_{\mathrm{At}} \mathrm{MOD}\right)^{20,21}$ (Fig. 1c). However, condensates in the $1 \mathrm{~A}_{\mathrm{At}} \mathrm{MOD}$ background were less punctate (Fig. 2a), which is consistent with the lower affinity of the modified native SSU for EPYC1 observed previously in yeast two-hybrid experiments ${ }^{19}$. Furthermore, we confirmed that condensates were formed when either EPYC1::tGFP or EPYC1::eGFP expression 
cassettes were transformed into S2 ${ }_{\mathrm{Cr}}$ individually (Extended Data Fig. 4a). Expression of a full length (i.e. non-truncated) variant of EPYC1-dGFP in Arabidopsis chloroplasts previously did not result in phase separation ${ }^{19}$, which was attributed to low levels of expression and an incompatible stoichiometry between EPYC1 and Rubisco, and possible proteolytic degradation. The results here indicate that the formation of a condensate is primarily due to the expression of the mature variant of EPYC1 rather than the level of expression per se, and that the required stoichiometry with Rubisco is achievable in planta. Furthermore, the observed reduction in proteolytic degradation may be accounted for by sequestration of EPYC1 within

111 a phase-separated compartment (Fig. 1b), which would be less accessible to large protease complexes $^{25}$.

113 Next we tested if the condensates exhibited internal mixing characteristics consistent with

114 the liquid-like behavior of pyrenoids (Fig. 2b, 2c). Fluorescence recovery after photobleaching 115 (FRAP) assays showed that condensates in live leaf cells had similar or increased rates of re116 homogenisation of EPYC1-dGFP (i.e. full recovery after 20-40 sec) compared to previous in 117 vitro $^{7}$ and in alga ${ }^{5}$ reports. The more rapid interchange in condensates compared to 118 Chlamydomonas pyrenoids may be due to the relatively reduced availability of EPYC1 binding 119 sites on Rubisco in the S2 ${ }_{\text {Cr }}$ plant-algal hybrid Rubisco background ${ }^{4}$. In contrast, condensates 120 in leaf tissue chemically cross-linked with formaldehyde showed no recovery after 121 photobleaching, which is consistent with that observed for Chlamydomonas pyrenoids ${ }^{5}$.

To test for the presence of Rubisco, the condensates were extracted from leaf tissue and examined by immunoblot. The condensates could be pelleted by gentle centrifugation ${ }^{6}$, and isolated condensates were shown to be enriched in EPYC1-dGFP and Rubisco (Fig. 3a). Protein samples were probed with either a polyclonal Rubisco antibody with a greater specificity for higher plant LSU and SSUs, or an antibody raised against the RbcS2 SSU isoform of Chlamydomonas $(\mathrm{CrRbcS} 2)$. Comparison of the resulting immunoblot data showed enrichment of the Chlamydomonas SSU in the pelleted condensate compared to native SSUs. Subsequent Coomassie staining of denatured, gel-separated extracts revealed that nearly half (49\%) of Rubisco in the initial extract contained Chlamydomonas SSU, while $82 \%$ of Rubisco in the pelleted condensate contained Chlamydomonas SSU (Fig. 3b). Pelleted condensates coalesced into larger droplets when resuspended, as expected for the liquid-like behavior of

133 EPYC1-Rubisco interactions (Fig. 3c) ${ }^{7}$. Immunogold analysis of chloroplast TEM images from

134 S2 Cr plants expressing EPYC1-dGFP showed approximately half (54\%) of all Rubisco was 135 contained within the condensate (Fig. 3d, 3e, Extended Data Fig. 4b). Consistent with 136 Coomasie staining, $81 \%$ of Rubisco containing Chlamydomonas SSU was located in the 
condensate. Thus, condensation of Rubisco is strongly associated with Rubisco complexes bearing the Chlamydomonas SSU, which constituted approximately 50\% of the Rubisco pool.

139 The latter is consistent with the expected expression levels of plant-algal hybrid Rubisco in $140 \mathrm{~S} 2 \mathrm{Cr}^{21}$. It is currently unclear if Rubisco can form a heterogenous L8S8 complex with different 141 SSU isoforms, or if only a single SSU isoform is favoured during assembly ${ }^{26}$. Thus, it remains 142 unclear whether the Rubisco pool within the condensate was comprised of a mixture of 143 homogeneous Rubisco complexes, or those containing both Arabidopsis and Chlamydomonas SSUs.

145 We compared the growth performance of three separate T2 EPYC1-dGFP S2 $2_{\mathrm{Cr}}$ transgenic lines (Ep1-3) screened for the presence of condensates with their matching T2 azygous segregant $\mathrm{S} 2 \mathrm{Cr}$ lines where the EPYC1-dGFP transgene was absent (Az1-3). Plants were cultivated under light levels typical for Arabidopsis growth $\left(200 \mu \mathrm{mol}\right.$ photons $\left.\mathrm{m}^{-2} \mathrm{~s}^{-1}\right)$ or under higher light levels $\left(900 \mu \mathrm{mol}\right.$ photons $\left.\mathrm{m}^{-2} \mathrm{~s}^{-1}\right)$ where growth rates are more limited by Rubisco activity (Fig. 4a, 4b, Extended Data Fig. 5) ${ }^{27}$. Regardless of the growth conditions, rosette expansion rates or biomass accumulation were not distinguishable between transformants and their segregant controls. Similarly, T2 EPYC1-dGFP WT plants (EpWT) showed no significant differences compared to T2 segregant lines (AzWT). Due to the reduced Rubisco content in the $\mathrm{S} 2 \mathrm{Cr}$ background and differences in Rubisco catalytic characteristics, the growth performance of $\mathrm{S} 2 \mathrm{Cr}$ lines was slightly decreased compared to WT plants. The observed differences in growth were in line with those reported previously for $\mathrm{S} 2 \mathrm{Cr}$ and WT plants in the absence of EPYC1 ${ }^{21}$.

Photosynthetic parameters derived from response curves of $\mathrm{CO}_{2}$ assimilation rate to the intercellular $\mathrm{CO}_{2}$ concentration under saturating light were similar between respective Ep and

160 Az lines (Fig. 4c, 4d, Extended Data Table 1). The presence of condensates did not influence 161 the maximum achievable rates of Rubisco carboxylation $\left(V_{\mathrm{cmax}}\right)$. Notably, the $\mathrm{CO}_{2}$ assimilation 162 rates at ambient concentrations of $\mathrm{CO}_{2}$ for $\mathrm{Ep}$ and $\mathrm{Az}$ lines were comparable to WT lines when 163 normalized for Rubisco content $\left(A_{\text {Rubisco }}\right)$ (Fig. 4d). This suggests that the modest reductions in 164 Rubisco turnover rate $\left(k_{\mathrm{cat}}{ }^{\mathrm{c}}\right)$ and specificity $\left(S_{\mathrm{C} / \mathrm{O}}\right)$ for the plant-algal hybrid Rubisco in $\mathrm{S} 2 \mathrm{Cr}$ compared to WT plants have only a mild impact on the efficiency of photosynthetic $\mathrm{CO}_{2}$ assimilation and that the observed differences in growth rates are more associated with the reduced levels of Rubisco in S2 Cr plants $^{21}$. Mesophyll conductance $\left(g_{\mathrm{m}}\right)$ levels were also reduced in all $\mathrm{S} 2 \mathrm{Cr}$ lines compared to WT plants, which is consistent with the impact of reduced

169 Rubisco content on $g_{\mathrm{m}}$ observed in transplastomic tobacco ${ }^{28}$. Condensate formation in the $1701 \mathrm{~A}_{\mathrm{At}} \mathrm{MOD}$ background, where catalytic characteristics of the hybrid Rubisco are 
indistinguishable from that of WT Rubisco, indicates that the SSU can be further engineered to optimise phase separation, and Rubisco content and performance (Fig. 1c) ${ }^{19}$. Measurements

173 of the maximum electron transport rate $\left(J_{\max }\right)$ and the maximum potential quantum efficiency 174 of Photosystem II $\left(F_{\mathrm{v}} / F_{\mathrm{m}}\right)$ were also indistinguishable between transformant and segregant 175 lines (Extended Data Table 1). Thus, the apparent displacement of the thylakoid membrane 176 matrix by the condensates (Fig. 1e) had no obvious impact on the efficiency of the light 177 reactions of photosynthesis.

178 Our findings show that EPYC1 and specific residues on the SSU are sufficient to aggregate 179 Rubisco into a single proto-pyrenoid condensate, which has no apparent negative impact on 180 plant growth. The overall photosynthetic performances of $\mathrm{S} 2 \mathrm{Cr}$ transgenic lines appeared unaffected by the condensate, which suggests that conditions inside higher plant chloroplasts are highly compatible with the presence of pyrenoid-type bodies. Our data is arguably the key step for the assembly of a pyrenoid-based CCM into plants that could increase crop yield potentials by $>60 \%$ (Fig. $4 \mathrm{e})^{8,9,29}$. Previously described approaches for engineering the cyanobacterial carboxysome-based CCM require engineering of the chloroplast-encoded Rubisco large subunit, which is not generally feasible in major grain crops such as wheat and rice $^{30}$, although expression of the large subunit from the nucleus of maize plants has been recently demonstrated ${ }^{31}$. Furthermore, transgenic plants expressing cyanobacterial Rubisco require high concentrations of $\mathrm{CO}_{2}$ to grow. Here we demonstrate that condensation of Rubisco is achievable through modification of the nuclear-encoded SSU, which is significantly more amenable to genetic modification. Future work will focus on progressing towards a minimal functional algal CCM by tethering the condensate to a thylakoid membrane for bicarbonate delivery using appropriate transporters ${ }^{32-34}$, as well as introduction of a lumenal carbonic anhydrase and a stromal $\mathrm{CO}_{2}$ salvage system ${ }^{35-37}$.

\section{Methods}

\section{Plant material and growth conditions}

198 Arabidopsis (Arabidopsis thaliana, Col-0 background) seeds were sown on compost, stratified for $3 \mathrm{~d}$ at $4{ }^{\circ} \mathrm{C}$ and grown at $20^{\circ} \mathrm{C}$, ambient $\mathrm{CO}_{2}$ and $70 \%$ relative humidity under either 200 or $900 \mu \mathrm{mol}$ photons $\mathrm{m}^{-2} \mathrm{~s}^{-1}$ supplied by cool white LED lights (Percival SE-41AR3cLED, CLF different genotypes, plants were grown from seeds of the same age and storage history, harvested from plants grown in the same environmental conditions. 


\section{Construct design and transformation}

206 The coding sequence of EPYC1 was codon optimised for expression in higher plants as in

207 Atkinson et al. ${ }^{19}$. Mature EPYC1 was cloned directly into the level 0 acceptor vector 208 pAGM1299 of the Plant MoClo system ${ }^{38}$. To generate fusion proteins, gene expression 209 constructs were assembled into binary level 2 acceptor vectors. The 35S cauliflower mosaic 210 virus (CaMV) promoter and CsVMV (cassava vein mosaic virus) promoter were used to drive 211 expression. Level 2 vectors were transformed into Agrobacterium tumefaciens (AGL1) for stable insertion in Arabidopsis plants by floral dipping ${ }^{39}$. Homozygous transgenic and azygous lines were identified in the T2 generation using the pFAST-R selection cassette ${ }^{40}$.

\section{Protein analyses}

216 Soluble protein was extracted from frozen leaf material of 21-d-old plants (sixth and seventh 217 leaf) in protein extraction buffer (50mM HEPES-KOH pH 7.5 with $17.4 \%$ glycerol, $2 \%$ Triton 218 X-100 and cOmplete Mini EDTA-free Protease Inhibitor Cocktail (Roche, Basel, Switzerland). 219 Samples were heated at $70{ }^{\circ} \mathrm{C}$ for 15 min with 1 x Bolt LDS sample buffer (ThermoFisher Scientific, UK) and $200 \mathrm{mM}$ DTT. Extracts were centrifuged and the supernatants subjected to SDS-PAGE on a $12 \%(\mathrm{w} / \mathrm{v})$ polyacrylamide gel and transferred to a nitrocellulose membrane. Membranes were probed with rabbit serum raised against wheat Rubisco at 1:10,000 dilution ${ }^{41}$, the SSU RbcS2 from Chlamydomonas $(\mathrm{CrRbcS} 2)$ (raised to the C-terminal region of the SSU (KSARDWQPANKRSV) by Eurogentec, Southampton, UK) at 1:1,000 dilution, ACTIN (66009-1-Ig, Proteintech, UK) at 1:1000 dilution and EPYC1 at 1:2,000 dilution ${ }^{6}$, followed by IRDye 800CW goat anti-rabbit IgG (LI-COR Biotechnology, Cambridge, UK) at 1:10,000 dilution, and visualised using the Odyssey CLx imaging system (LI-COR Biotechnology).

\section{qRT-PCR analysis}

230 Total RNA was isolated from leaves of 21-d-old plants (as above) using the RNeasy plant mini

231 kit (Qiagen, USA). Isolated RNA was treated with DNase (Qiagen, USA) and reverse transcribed with random primers using the GoScript Reverse Transcription kit (Promega, USA). Reverse transcription quantitative PCR (RT-qPCR) was carried out with Takyon No ROX SYBR mastermix (Eurogentec, Belgium) as in Atkinson et al. ${ }^{17}$, and run on a LightCycler 480 (Roche, Switzerland). Gene-specific primers were designed to amplify either eGFP (5'CAGATTACGCCGTGAGCAAG-3' and 5'-GCTGAACTTGTGGCCGTTTA-3') or tGFP (5'-GCATCAGGGGTCTTGAAAGC-3' and 5'-TCCTTCAAAACGGTGGACCT-3') (IDT, 
pair. Two reference genes At4g26410 (RHIP1) and At1g13320 (PP2A) were used for normalization $^{42}$. The relative expression of eGFP and tGFP was calculated according to Vandesompele et al. ${ }^{43}$.

\section{Condensate extraction}

244 Soluble protein was extracted as before, then filtered through Miracloth (Merck Millipore, 245 Burlington, Massachusetts, USA), and centrifuged at $500 \mathrm{~g}$ for $3 \mathrm{~min}$ at $4{ }^{\circ} \mathrm{C}$, as in Mackinder et al. ${ }^{6}$. The pellet was discarded, and the extract centrifuged again for $12 \mathrm{~min}$. The resulting pellet was washed once in protein extraction buffer, then re-suspended in a small volume of buffer and centrifuged again for $5 \mathrm{~min}$. Finally, the pellet was re-suspended in $25 \mu \mathrm{l}$ of extraction buffer and used in confocal analysis or SDS-PAGE electrophoresis.

\section{Growth analysis and photosynthetic measurements}

252 Rosette growth rates were quantified using an in-house imaging system ${ }^{44}$. Maximum quantum yield of photosystem II (PSII) $\left(F_{\mathrm{v}} / F_{\mathrm{m}}\right)$ was measured on 32-d-old plants using a Hansatech

254 Handy PEA continuous excitation chlorophyll fluorimeter (Hansatech Instruments Ltd, King's Lynn, UK $)^{5}$. Gas exchange and chlorophyll fluorescence were determined using a LI-COR LI6400 (LI-COR, Lincoln, Nebraska, USA) portable infra-red gas analyser with a 6400-40 leaf chamber on either the sixth or seventh leaf of 35- to 45-d-old non-flowering rosettes grown in large pots under $200 \mu \mathrm{mol}$ photons $\mathrm{m}^{-2} \mathrm{~s}^{-1}$ to generate leaf area sufficient for gas exchange measurements ${ }^{46}$. The response of $A$ to the intercellular $\mathrm{CO}_{2}$ concentration $\left(C_{\mathrm{i}}\right)$ was measured at various $\mathrm{CO}_{2}$ concentrations $(50,100,150,200,250,300,350,400,600,800,1000$ and $\left.1200 \mu \mathrm{mol} \mathrm{mol}^{-1}\right)$ under saturating light $\left(1,500 \mu \mathrm{mol}\right.$ photons $\left.\mathrm{m}^{-2} \mathrm{~s}^{-1}\right)$. For all gas exchange experiments, the flow rate was kept at $200 \mu \mathrm{mol} \mathrm{mol}{ }^{-1}$, leaf temperature was controlled at $25^{\circ} \mathrm{C}$ and chamber relative humidity was maintained at $c a .70 \%$. Measurements were performed after net assimilation and stomatal conductance had reached steady state. Gas exchange data were corrected for $\mathrm{CO}_{2}$ diffusion from the measuring chamber as in Bellasio et al. ${ }^{47}$. To estimate $V_{\text {cmax }}, J_{\max }, A_{\text {Rubisco }}$, the $\mathrm{CO}_{2}$ compensation point $\left(\ulcorner)\right.$ and mesophyll conductance to $\mathrm{CO}_{2}\left(g_{\mathrm{m}}\right)$ the $A / C$ i data were fitted to the $\mathrm{C}_{3}$ photosynthesis model as in Ethier and Livingston ${ }^{48}$ using the $25{ }^{\circ} \mathrm{C}$ and the Rubisco content of WT and $\mathrm{S} 2 \mathrm{Cr}$ lines ${ }^{21}$.

\section{Confocal laser scanning and fluorescence recovery after photobleaching (FRAP)}


272 Leaves were imaged with a Leica TCS SP8 laser scanning confocal microscope (Leica 273 Microsystems, Milton Keynes, UK) as in Atkinson et al. ${ }^{17}$. Processing of images was done with

274 Leica LAS AF Lite software. Condensate and chloroplast dimensions were measured from 275 confocal images using Fiji (ImageJ, v1.52n) ${ }^{49}$. Condensate volume was calculated as a sphere, 276 while chloroplast volume was calculated as an ellipsoid, where depth was estimated as $25 \%$ of 277 the measured width. Chloroplast volumes varied between $24-102 \mu \mathrm{m}^{3}$, which was within the 278 expected size range and distribution for Arabidopsis chloroplasts ${ }^{50}$. Comparative measurements of pyrenoid area were performed using Fiji on TEM cross-section images of WT C. reinhardtii cells (cMJ030) as described in Itakura et al. ${ }^{23}$. FRAP was carried out on live leaf tissue and tissue that had been fixed by infiltrating with $4 \%(\mathrm{v} / \mathrm{v})$ formaldehyde for $90 \mathrm{~min}$ in a vacuum chamber, then infiltrating three times for 5 min each with phosphate-buffered saline (PBS) at $\mathrm{pH}$ 7.4. The SP8 microscope was used with a 40x water immersion objective and a photomultiplier tube (PMT) detector. The $488 \mathrm{~nm}$ laser was set to $2 \%$ power for pre- and postbleach images, and 25\% for the bleaching step. Pre-bleach images were captured at $220 \mathrm{~ms}$ intervals (6 in total) and post-bleach images were captured at $400 \mathrm{~ms}$ intervals (at least 120). For photo-bleaching the laser was directed to a region with a diameter of $0.5-0.6 \mu \mathrm{m}$ on one side of the EPYC1 aggregate. Recovery time was calculated by comparing GFP expression to an unbleached region of the same size.

\section{Super-resolution image microscopy}

292 Super-resolution images were acquired using structured illumination microscopy. Samples were prepared on high precision cover-glass (Zeiss, Jena, Germany). 3D SIM images were acquired on a N-SIM (Nikon Instruments, UK) using a 100x 1.49NA lens and refractive index matched immersion oil (Nikon Instruments). Samples were imaged using a Nikon Plan Apo TIRF objective (NA 1.49, oil immersion) and an Andor DU-897X-5254 camera using a 488nm laser line. Z-step size for z stacks was set to $0.120 \mu \mathrm{m}$ as required by manufacturer's software. For each focal plane, 15 images (5 phases, 3 angles) were captured with the NIS-Elements software. SIM image processing, reconstruction and analysis were carried out using the N-SIM module of the NIS-Element Advanced Research software. Images were checked for artefacts using the SIMcheck software (www.micron.ox.ac.uk/software/SIMCheck.php). Images were reconstructed using NiS Elements software v4.6 (Nikon Instruments) from a z stack comprising of no less than $1 \mu \mathrm{m}$ of optical sections. In all SIM image reconstructions the Wiener and Apodization filter parameters were kept constant. 


\section{Immunogold labelling and electron microscopy}

307 Leaf samples were taken from 21-d-old S2 Cr plants and S2 $\mathrm{Cr}$ transgenic lines expressing 308 EPYC1-dGFP and fixed with with 4\% (v/v) paraformaldehyde, 0.5\% (v/v) glutaraldehyde and $3090.05 \mathrm{M}$ sodium cacodylate ( $\mathrm{pH}$ 7.2). Leaf strips (1 mm wide) were vacuum infiltrated with 310 fixative three times for $15 \mathrm{~min}$, then rotated overnight at $4^{\circ} \mathrm{C}$. Samples were rinsed three times 311 with PBS ( $\mathrm{pH} 7.4$ ) then dehydrated sequentially by vacuum infiltrating with $50 \%, 70 \%, 80 \%$ 312 and $90 \%$ ethanol (v/v) for $1 \mathrm{hr}$ each, then three times with 100\%. Samples were infiltrated with 313 increasing concentrations of LR White Resin $(30 \%, 50 \%, 70 \%$ [w/v]) mixed with ethanol for $3141 \mathrm{hr}$ each, then $100 \%$ resin three times. The resin was polymerised in capsules at $50^{\circ} \mathrm{C}$ 315 overnight. Sections ( $1 \mu \mathrm{m}$ thick) were cut on a Leica Ultracut ultramicrotome, stained with 316 Toluidine Blue, and viewed in a light microscope to select suitable areas for investigation.

317 Ultrathin sections (60 nm thick) were cut from selected areas and mounted onto plastic-coated 318 copper grids. Grids were blocked with 1\% (w/v) BSA in TBSTT (Tris-buffered saline with $3190.05 \%[\mathrm{v} / \mathrm{v}]$ Triton X-100 and $0.05 \%[\mathrm{v} / \mathrm{v}]$ Tween 20), incubated overnight with anti-Rubisco antibody in TBSTT at 1:250 dilution or anti-CrRbcS2 antibody at 1:50 dilution, and washed twice each with TBSTT and water. Incubation with $15 \mathrm{~nm}$ gold particle-conjugated goat antirabbit secondary antibody (Abcam, Cambridge, UK) in TBSTT was carried out for $1 \mathrm{hr}$ at 1:200 dilution for Rubisco labelling or 1:10 for CrRbcS2 labelling, before washing as before. Grids were stained in 2\% (w/v) uranyl acetate then viewed in a JEOL JEM-1400 Plus TEM (JEOL, Peabody, Massachusetts, USA). Images were collected on a GATAN OneView camera (GATAN, Pleasanton, California, USA).

\section{Statistical analyses}

Results were subjected to analysis of variance (ANOVA) to determine the significance of the difference between sample groups. When ANOVA was performed, Tukey's honestly significant difference (HSD) post-hoc tests were conducted to determine the differences between the individual treatments (IBM SPSS Statistics Ver. 26.0, Chicago, IL, USA).

\section{Data availability}

All relevant data and plant materials are available from the authors upon request. Raw data corresponding to the figures and results described in this manuscript are available online [Edinburgh DataShare address to be added]. Additional data reported in this paper are presented as Extended Data. 


\section{Acknowledgements}

This work was funded by the UK Research and Innovation Biotechnology and Biological Sciences Research Council (BB/S015531/1) and Leverhulme Trust (RPG-2017-402). The authors thank Moritz Meyer (Princeton University) for providing additional TEM cross-section images of Chlamydomonas and Ann Wheeler of ESRIC (Edinburgh Super-Resolution Imaging Consortium) for expertise and assistance with SIM. TEM was carried out with the support of Stephen Mitchell and the Wellcome Trust Multi User Equipment Grant (WT104915MA).

\section{References}

1 Andersson, I. \& Backlund, A. Structure and function of Rubisco. Plant Physiol Bioch 46, 275-291, doi:10.1016/j.plaphy.2008.01.001 (2008).

2 Salesse-Smith, C. E. et al. Overexpression of Rubisco subunits with RAF1 increases Rubisco content in maize. Nat Plants 4, 802-810, doi:10.1038/s41477-018-0252-4 (2018).

3 Flamholz, A. I. et al. Revisiting Trade-offs between Rubisco Kinetic Parameters. Biochemistry-Us 58, 3365-3376, doi:10.1021/acs.biochem.9b00237 (2019).

4 Mackinder, L. C. M. et al. A Spatial Interactome Reveals the Protein Organization of the Algal CO2-Concentrating Mechanism. Cell 171, 133-147 e114, doi:10.1016/j.cell.2017.08.044 (2017).

5 Freeman Rosenzweig, E. S. et al. The Eukaryotic CO2-Concentrating Organelle Is Liquid-like and Exhibits Dynamic Reorganization. Cell 171, 148-162 e119, doi:10.1016/j.cell.2017.08.008 (2017).

6 Mackinder, L. C. et al. A repeat protein links Rubisco to form the eukaryotic carbonconcentrating organelle. Proceedings of the National Academy of Sciences of the United States of America 113, 5958-5963, doi:10.1073/pnas.1522866113 (2016).

7 Wunder, T., Cheng, S. L. H., Lai, S. K., Li, H. Y. \& Mueller-Cajar, O. The phase separation underlying the pyrenoid-based microalgal Rubisco supercharger. Nature communications 9, 5076, doi:10.1038/s41467-018-07624-w (2018).

8 McGrath, J. M. \& Long, S. P. Can the cyanobacterial carbon-concentrating mechanism increase photosynthesis in crop species? A theoretical analysis. Plant physiology 164, 2247-2261, doi:10.1104/pp.113.232611 (2014).

9 Long, S. P., Burgess, S., Causton, I. in Sustaining Global Food Security: The Nexus of Science and Policy. (ed R. S. Zeigler) Ch. 9, (CSIRO Publishing, 2019).

10 Galmes, J. et al. Expanding knowledge of the Rubisco kinetics variability in plant species: environmental and evolutionary trends. Plant Cell and Environment 37, 19892001, doi:10.1111/pce.12335 (2014).

11 Young, J. N. et al. Large variation in the Rubisco kinetics of diatoms reveals diversity among their carbon-concentrating mechanisms. $J$ Exp Bot 67, 3445-3456, doi:10.1093/jxb/erw163 (2016).

12 Bathellier, C., Tcherkez, G., Lorimer, G. H. \& Farquhar, G. D. Rubisco is not really so bad. Plant Cell and Environment 41, 705-716, doi:10.1111/pce.13149 (2018).

13 Wilson, R. H., Whitney, S. M. in Directed Enzyme Evolution: Advances and Applications (ed M. Alcalde) Ch. 4, 101-126 (Springer International Publishing AG, 2017). 
14 Long, S. P., Marshall-Colon, A. \& Zhu, X. G. Meeting the global food demand of the future by engineering crop photosynthesis and yield potential. Cell 161, 56-66, doi:10.1016/j.cell.2015.03.019 (2015).

15 Li, F. W. et al. Anthoceros genomes illuminate the origin of land plants and the unique biology of hornworts. Nat Plants 6, 259-272, doi:10.1038/s41477-020-0618-2 (2020).

16 Raven, J. A., Beardall, J. \& Sanchez-Baracaldo, P. The possible evolution and future of CO2-concentrating mechanisms. Journal of Experimental Botany 68, 3701-3716, doi:10.1093/jxb/erx110 (2017).

17 Atkinson, N. et al. Introducing an algal carbon-concentrating mechanism into higher plants: location and incorporation of key components. Plant biotechnology journal 14, 1302-1315, doi:10.1111/pbi.12497 (2016).

18 Rae, B. D. et al. Progress and challenges of engineering a biophysical CO2concentrating mechanism into higher plants. $J$ Exp Bot 68, 3717-3737, doi:10.1093/jxb/erx133 (2017).

19 Atkinson, N. et al. The pyrenoidal linker protein EPYC1 phase separates with hybrid Arabidopsis-Chlamydomonas Rubisco through interactions with the algal Rubisco small subunit. J Exp Bot 70, 5271-5285, doi:10.1093/jxb/erz275 (2019).

20 Meyer, M. T. et al. Rubisco small-subunit alpha-helices control pyrenoid formation in Chlamydomonas. Proceedings of the National Academy of Sciences of the United States of America 109, 19474-19479, doi:10.1073/pnas.1210993109 (2012).

21 Atkinson, N. et al. Rubisco small subunits from the unicellular green alga Chlamydomonas complement Rubisco-deficient mutants of Arabidopsis. The New phytologist 214, 655-667, doi:10.1111/nph.14414 (2017).

22 Wunder, T., Guo Oh, Z. \& Mueller-Cajar, O. $\mathrm{CO}_{2}$-fixing liquid droplets: Towards a dissection of the microalgal pyrenoid. Traffic 20, 380-389, doi: 10.1111/tra.12650 (2019).

23 Itakura, A. K. et al. A Rubisco-binding protein is required for normal pyrenoid number and starch sheath morphology in Chlamydomonas reinhardtii. Proceedings of the National Academy of Sciences of the United States of America 116, 18445-18454, doi:10.1073/pnas.1904587116 (2019).

24 Rosowski, K. A et al. Elastic ripening and inhibition of liquid-liquid phase separation. Nature Physics 16, $422-425$ (2020).

25 van der Hoorn, R. A. L. \& Rivas, S. Unravelling the mode of action of plant proteases. The New phytologist 218, 879-881, doi:10.1111/nph.15156 (2018).

26 Valegård, K., Hasse, D., Andersson, I. \& Gunn, L. Structure of Rubisco from Arabidopsis thaliana in complex with 2-carboxyarabinitol-1,5-bis-phosphate. Acta Crystallographica Section D-Structural Biology 74, 1-9 (2018).

27 Lauerer, M. et al. Decreased ribulose-1,5-bisphosphate carboxylase-oxygenase in transgenic tobacco transformed with "antisense" rbcS. VI. Effect on photosynthesis in plants grown at different irradiance. Planta 190, 332-345, doi:10.1007/bf00196962 (1993).

28 Galmes, J., Perdomo, J. A., Flexas, J. \& Whitney, S. M. Photosynthetic characterization of Rubisco transplantomic lines reveals alterations on photochemistry and mesophyll conductance. Photosynthesis Research 115, 153-166, doi:10.1007/s11120-013-9848-8 (2013).

29 Price, G. D., Badger, M. R. \& von Caemmerer, S. The prospect of using cyanobacterial bicarbonate transporters to improve leaf photosynthesis in C3 crop plants. Plant physiology 155, 20-26, doi:10.1104/pp.110.164681 (2011).

30 Long, B. M. et al. Carboxysome encapsulation of the CO2-fixing enzyme Rubisco in tobacco chloroplasts. Nature communications 9, doi:Artn 3570 
10.1038/S41467-018-06044-0 (2018).

31 Salesse-Smith, C., et al. Overexpression of Rubisco subunits with RAF1 increases Rubisco content in maize. Nature Plants 4, 802-810, doi: 10.1038/s41477-018-0252-4 (2018).

32 Engel, B. D. et al. Native architecture of the Chlamydomonas chloroplast revealed by in situ cryo-electron tomography. eLife 4, doi: e0488910.7554/eLife.04889 (2015).33 Yamano, T., Sato, E., Iguchi, H., Fukuda, Y. \& Fukuzawa, H. Characterization of cooperative bicarbonate uptake into chloroplast stroma in the green alga Chlamydomonas reinhardtii. Proceedings of the National Academy of Sciences of the United States of America 112, 7315-7320, doi:10.1073/pnas.1501659112 (2015).

34 Mukherjee, A. et al. Thylakoid localized bestrophin-like proteins are essential for the $\mathrm{CO} 2$ concentrating mechanism of Chlamydomonas reinhardtii. Proceedings of the National Academy of Sciences of the United States of America 116, 16915-16920, doi:10.1073/pnas.1909706116 (2019).

35 Karlsson, J. et al. A novel alpha-type carbonic anhydrase associated with the thylakoid membrane in Chlamydomonas reinhardtii is required for growth at ambient $\mathrm{CO} 2$. The EMBO journal 17, 1208-1216, doi:10.1093/emboj/17.5.1208 (1998).

36 Yamano, T. et al. Light and low-CO2-dependent LCIB-LCIC complex localization in the chloroplast supports the carbon-concentrating mechanism in Chlamydomonas reinhardtii. Plant \& cell physiology 51, 1453-1468, doi:10.1093/pcp/pcq105 (2010).

37 Toyokawa, C., Y. T., Fukuzawa H. Pyrenoid starch sheath is required for LCIB localization and the CO2-concentrating mechanism in green algae. Plant physiology, doi:10.1104/pp.19.01587 (2020).

38 Engler, C. et al. A Golden Gate Modular Cloning Toolbox for Plants. Acs Synth Biol 3, 839-843, doi:10.1021/sb4001504 (2014).

39 Clough, S. J. \& Bent, A. F. Floral dip: a simplified method for Agrobacterium-mediated transformation of Arabidopsis thaliana. The Plant journal : for cell and molecular biology 16, 735-743 (1998).

40 Shimada, T. L., Shimada, T. \& Hara-Nishimura, I. A rapid and non-destructive screenable marker, FAST, for identifying transformed seeds of Arabidopsis thaliana. Plant Journal 61, 519-528, doi:10.1111/j.1365-313X.2009.04060.x (2010).

41 Howe, C. J. et al. Location and nucleotide sequence of the gene for the protontranslocating subunit of wheat chloroplast ATP synthase. Proceedings of the National Academy of Sciences of the United States of America 79, 6903-6907 (1982).

42 Czechowski, T., Stitt, M., Altmann, T., Udvardi, M. K. \& Scheible, W. R. Genomewide identification and testing of superior reference genes for transcript normalization in Arabidopsis. Plant Physiology 139, 5-17 (2005).

43 Vandesompele, J. et al. Accurate normalization of real-time quantitative RT-PCR data by geometric averaging of multiple internal control genes. Genome Biology 3, 7 (2002).

44 Dobrescu, A., Scorza, L. C. T., Tsaftaris, S. A. \& McCormick, A. J. A "Do-It-Yourself" phenotyping system: measuring growth and morphology throughout the diel cycle in rosette shaped plants. Plant methods 13, 95 (2017).

45 Maxwell, K. \& Johnson, G. N. Chlorophyll fluorescence--a practical guide. J Exp Bot 51, 659-668 (2000).

46 Flexas, J. et al. Mesophyll conductance to CO2 in Arabidopsis thaliana. The New phytologist 175, 501-511, doi:10.1111/j.1469-8137.2007.02111.x (2007).

47 Bellasio, C., Beerling, D. J. \& Griffiths, H. An Excel tool for deriving key photosynthetic parameters from combined gas exchange and chlorophyll fluorescence: theory and practice. Plant, cell \& environment, doi:10.1111/pce.12560 (2015). 
48 Ethier, G. J. \& Livingston, N. J. On the need to incorporate sensitivity to $\mathrm{CO} 2$ transfer conductance into the Farquhar-von Caemmerer-Berry leaf photosynthesis model. Plant, Cell and Environment 27, 137-153, doi:10.1111/j.1365-3040.2004.01140.x (2004).

49 Schindelin, J. et al. Fiji: an open-source platform for biological-image analysis. Nature methods 9, 676-682, doi:10.1038/nmeth.2019 (2012).

50 Crumpton-Taylor, M., Grandison, S., Png, K. M., Bushby, A. J. \& Smith, A. M. Control of starch granule numbers in Arabidopsis chloroplasts. Plant physiology 158, 905-916, doi:10.1104/pp.111.186957 (2012).

51 Diamos, A. G. \& Mason, H. S. Chimeric 3' flanking regions strongly enhance gene expression in plants. Plant biotechnology journal 16, 1971-1982, doi:10.1111/pbi.12931 (2018).

52 Sade, N. et al. Differential tissue-specific expression of NtAQP1 in Arabidopsis thaliana reveals a role for this protein in stomatal and mesophyll conductance of $\mathrm{CO} 2$ under standard and salt-stress conditions. Planta 239, 357-366, doi:10.1007/s00425- 


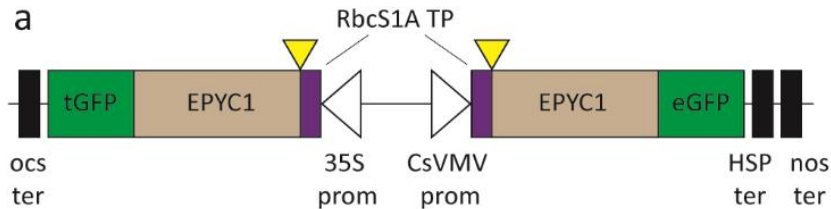

b
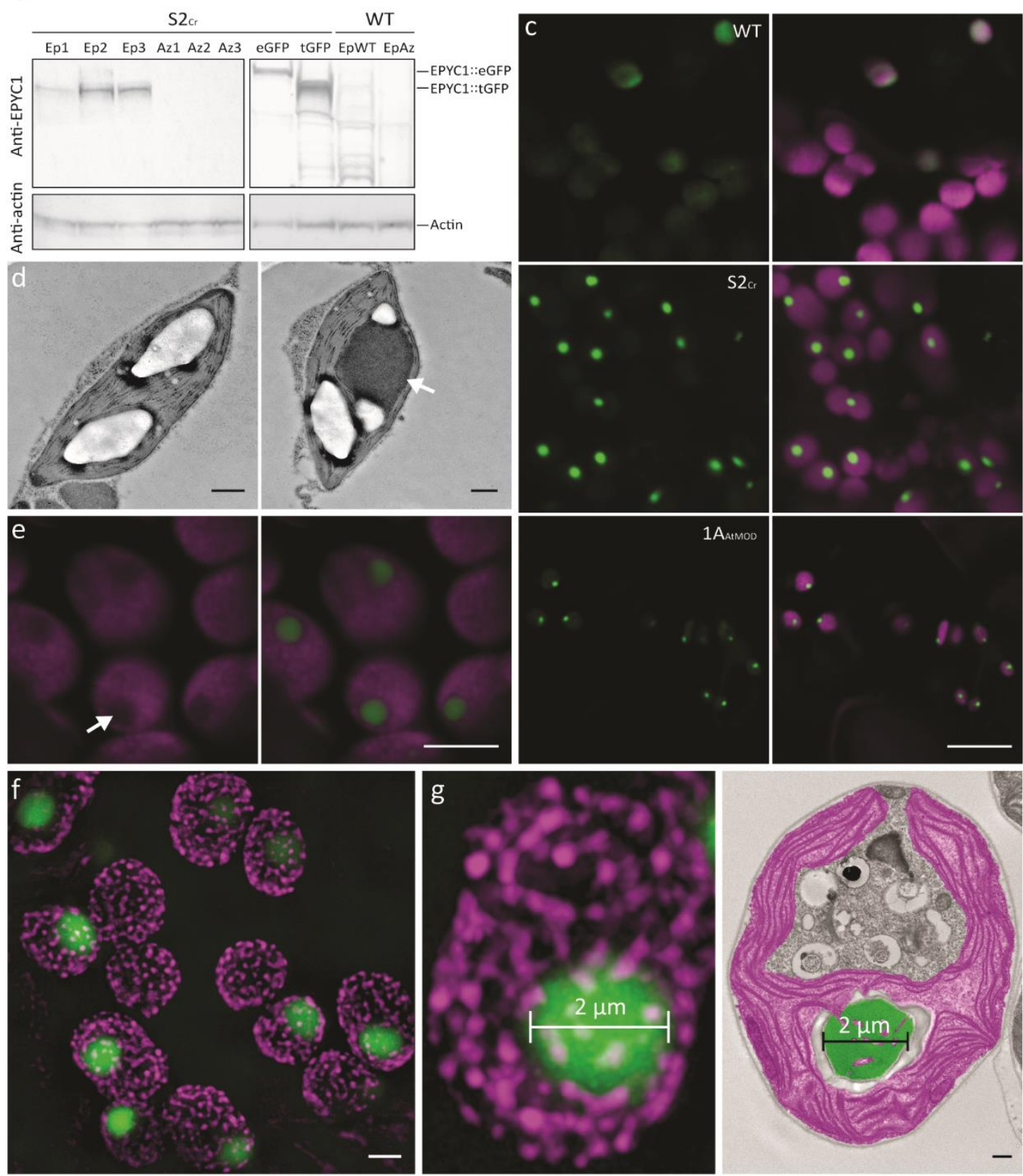

Figure 1. Expression of EPYC1 in the Arabidopsis line $\mathrm{S2} \mathrm{Cr}$ results in condensate

502 formation. a, Schematic representation of the dual GFP expression system (EPYC1-dGFP) for

503 EPYC1 truncated at amino acid residue 27 (as indicated by yellow triangles) and fused at the

504 N-terminus to the chloroplastic transit peptide (TP) sequence of the Arabidopsis Rubisco small 505 subunit RbcS1A. EPYC1 was further fused at the C-terminus to either enhanced GFP (eGFP) or turboGFP (tGFP), and driven by the 35S CaMV promoter (35S prom) or CsVMV promoter (CsVMV prom), respectively. For the latter expression cassette, a dual terminator system was 
508 used to increase expression ${ }^{51}$. b, EPYC1 protein levels in Arabidopsis plants as assessed by 509 immunoblot analysis with anti-EPYC1 antibodies. Shown are three T2 S2 $\mathrm{Cr}$ transgenic plants

510 expressing EPYC1-dGFP (Ep1-3) and azygous segregants (Az1-3), and S2Cr plants 511 transformed with only EPYC1::tGFP (55.4 kDa) or EPYC1::eGFP (63.9 kDa). Also displayed 512 are a T2 EPYC1-dGFP WT transformant (EpWT) and azygous segregant (AzWT). Anti-actin 513 is shown as a loading control underneath. c, Expression of EPYC1-dGFP in WT, S2 ${ }_{\mathrm{Cr}}$ and $5141 \mathrm{~A}_{\mathrm{At}} \mathrm{MOD}$ backgrounds. Green and purple signals are GFP and chlorophyll autofluorescence, 515 respectively. Overlapping signals are white. Scale bars $=10 \mu \mathrm{m}$. d, TEM images of chloroplasts 516 from $\mathrm{S} 2 \mathrm{Cr}$ plants with (right) and without (left) expression of EPYC1. A white arrowhead 517 indicates the dense dark grey area of the EPYC1 condensate. The large white structures are 518 starch granules. Scale bars $=0.5 \mu \mathrm{m}$. A representative chloroplast from a wild-type plant 519 expressing EPYC1-dGFP is shown for comparison in Extended Data Figure 3. e, Chlorophyll 520 autofluorescence is reduced at the site of EPYC1-dGFP accumulation (white arrow). Scale bar $521=5 \mu \mathrm{m}$. f, SIM microscopy showing EPYC1-dGFP condensates inside the chloroplast. The 522 magenta puncta show the position of grana stacks. Light magenta puncta indicate grana stacks 523 behind the condensate. Scale bar $=2 \mu \mathrm{m}$. g, Example comparison of condensate size (left, 2 $524 \mu \mathrm{m}$ ) with that of a pyrenoid in Chlamydomonas (right, representative TEM image highlighting 525 the pyrenoid in green and chloroplast in purple). Scale bar for TEM image $=0.5 \mu \mathrm{m}$. Images 526 of EPYC1-dGFP condensates in the $\mathrm{S} 2 \mathrm{Cr}$ background are from line Ep3. 
a

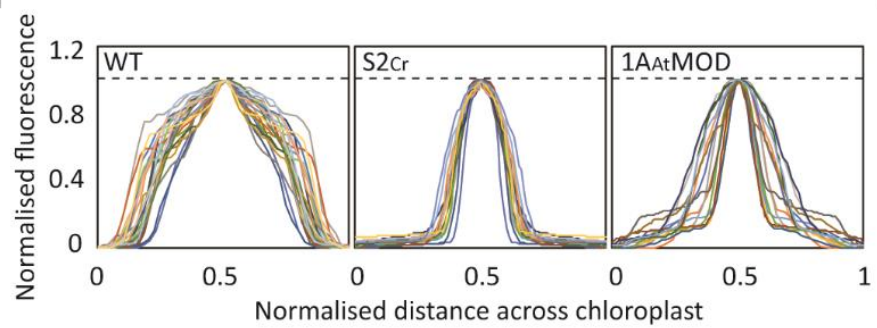

b

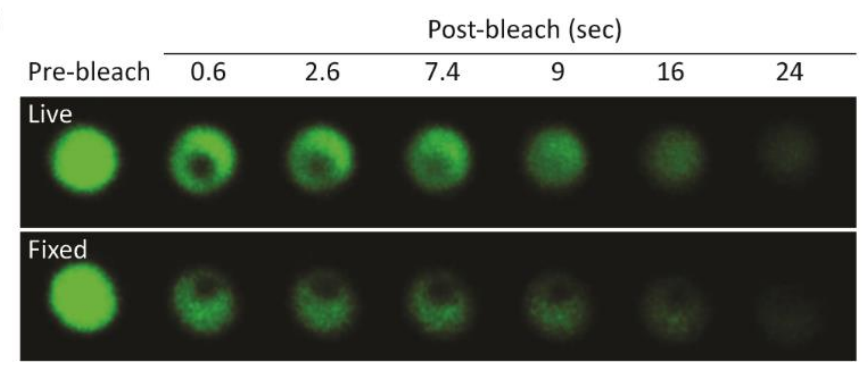

C

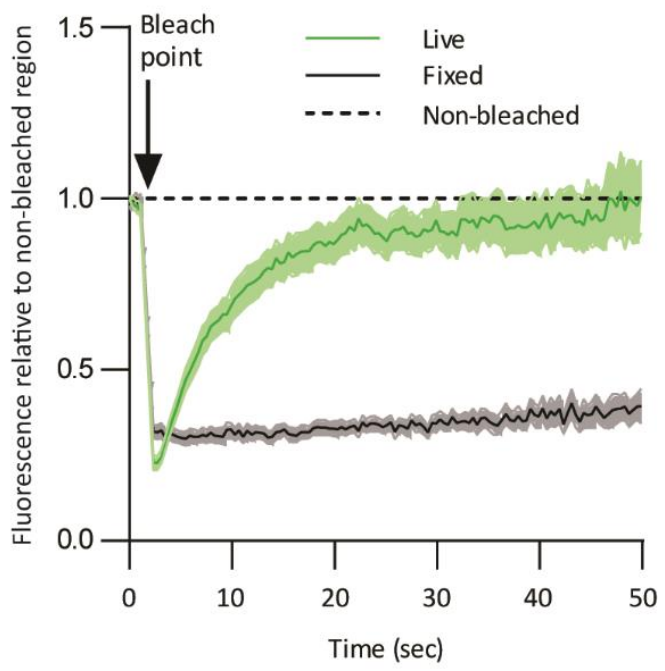

529 Figure 2. In planta condensates behave like liquid-liquid phase separated micro

530 compartments. a, Fluorescence distribution plots of EPYC1-dGFP across the chloroplast. The

531 intensity of the GFP fluorescence signal over the cross section of a chloroplast is shown in WT

$532(\mathrm{n}=28), \mathrm{S} 2 \mathrm{Cr}$ (line Ep3, n=17) and 1 $\mathrm{A}_{\mathrm{At}} \mathrm{MOD}(\mathrm{n}=22)$ backgrounds. Both GFP fluorescence and

533 cross section values have been normalised to 1 (as indicated by the dashed line), with the

534 highest value in the centre. b. Fluorescence recovery after photobleaching (FRAP) assays.

535 Condensates are shown from live (top) and fixed (bottom) leaf tissue from $\mathrm{S} 2{ }_{\mathrm{Cr}}$ transgenic line

536 Ep3 expressing EPYC1-dGFP. c, Fluorescence recovery of the bleached area in relation to the

537 non-bleached area of condensates. The mean and SEM are shown for 13-16 chloroplasts. 
a
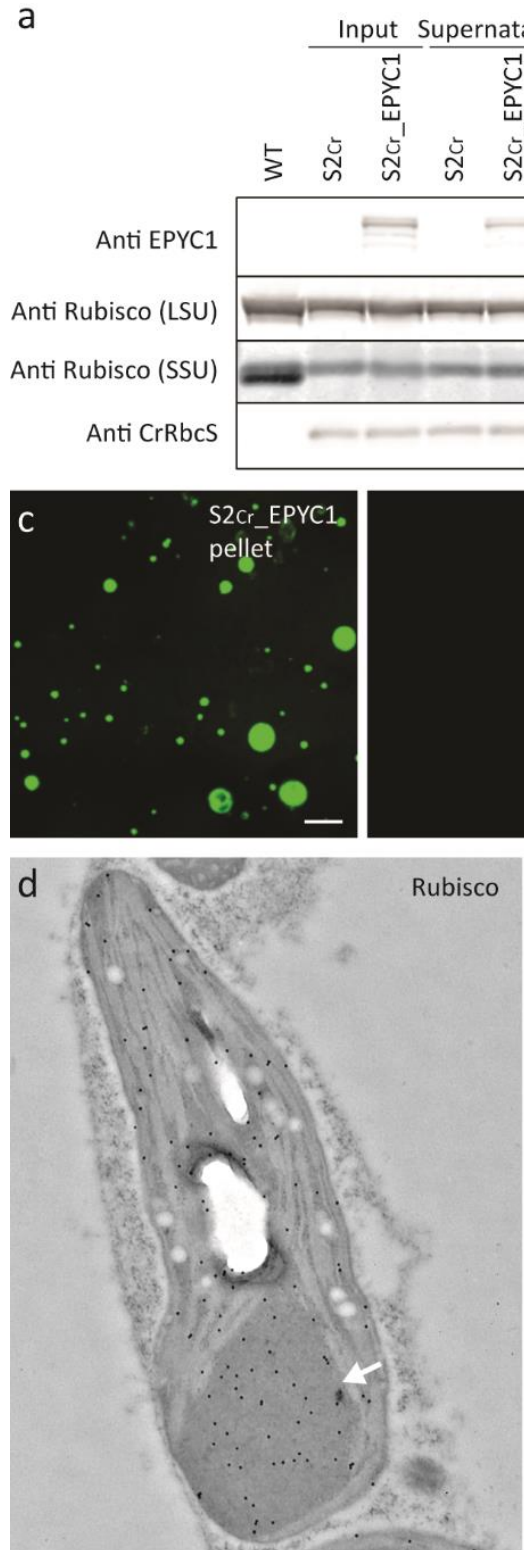

b

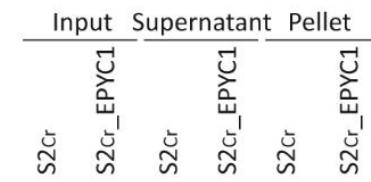

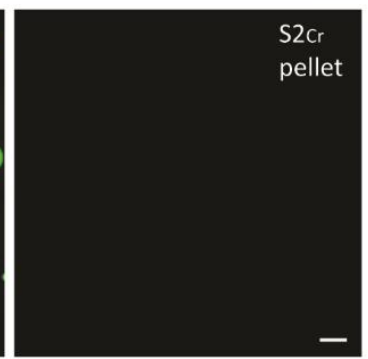

CrRbcS2

AtRbcS1B/2B/3B AtRbcS1A

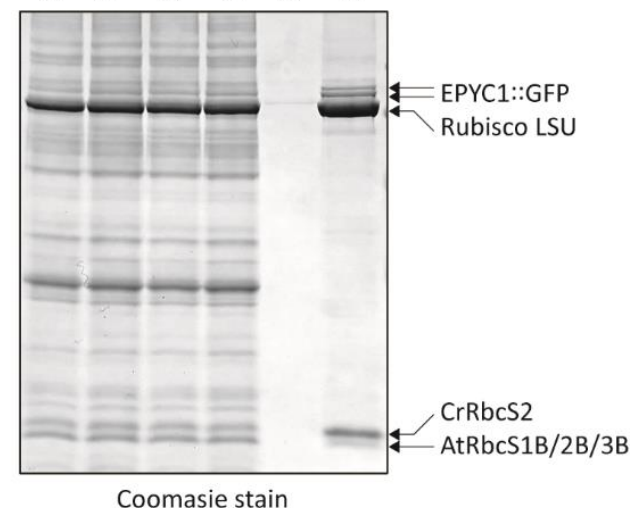

Coomasie stain

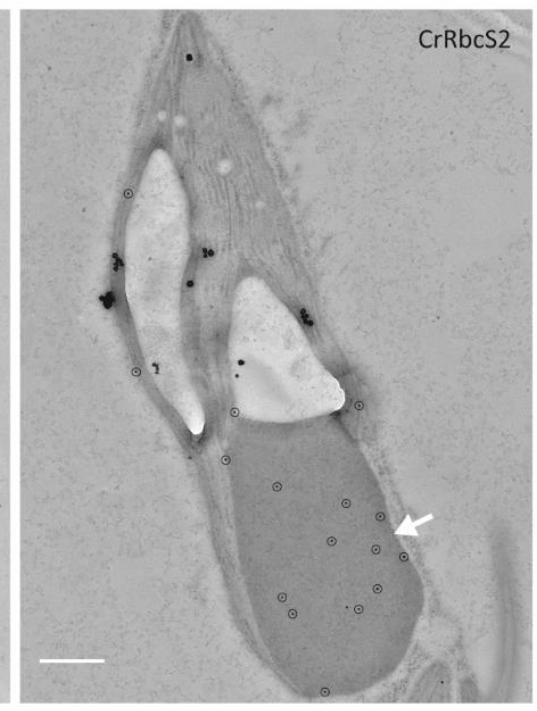

e

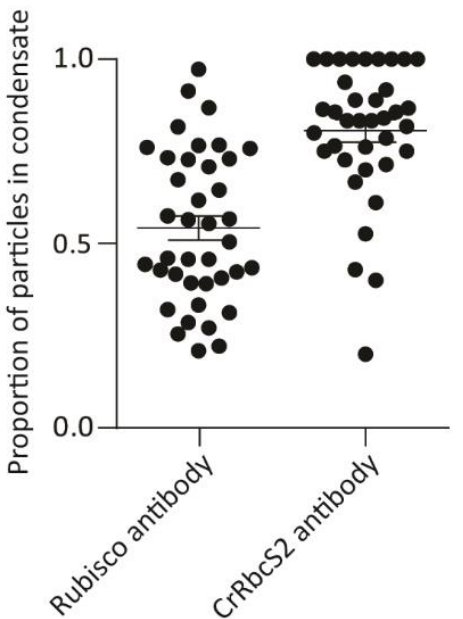

Figure 3. Condensates contain EPYC1 and plant-algal hybrid Rubisco. a, EPYC1 and

Rubisco protein levels in whole leaf tissue (input), the supernatant following condensate extraction and centrifugation (supernatant) and the pellet (pellet) as assessed by immunoblot analyses with anti-EPYC1, anti-Rubisco (LSU and SSU shown) or anti-CrRbcS2 antibodies.

544 Samples are shown for WT plants (WT), and S2 $2_{\mathrm{Cr}}$ mutants not expressing EPYC1 (S2 $\left.\mathrm{Cr}\right)$ and

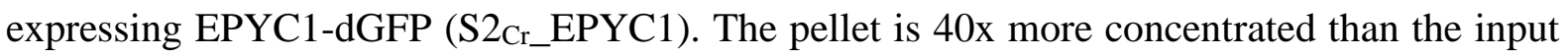
and supernatant. Molecular weights: LSU, 55 kD; RbcS1B, RbcS2B and RbcS3B, 14.8 kD; AtRbcS1A, 14.7kD; CrRbcS2 15.5 kD. b, Coomassie-stained SDS-PAGE gel showing the composition of the pelleted condensate. c, The condensates in the $\mathrm{S} 2_{\mathrm{Cr}}$ EPYC1 pellet coalescence to form large liquid droplets. Scale bar $=50 \mu \mathrm{m}$. d, Representative immunogold 
550 labelling of Rubisco in chloroplasts of an $\mathrm{S} 2 \mathrm{Cr}$ transgenic line Ep3 expressing EPYC1-dGFP

551 probed with polyclonal anti-Rubisco (left) or anti-CrRbcS2 (right) antibodies (dots are

552 highlighted for the latter). The condensates are marked by a white arrowhead. Large white

553 structures are starch granules. Scale bar $=0.5 \mu \mathrm{m}$. e, Proportion of gold nanoparticles inside

554 the condensate compared to the chloroplast for each antibody. The mean and SEM are shown

555 for 37-39 chloroplasts. Scale bar $=0.5 \mu \mathrm{m}$. 
a

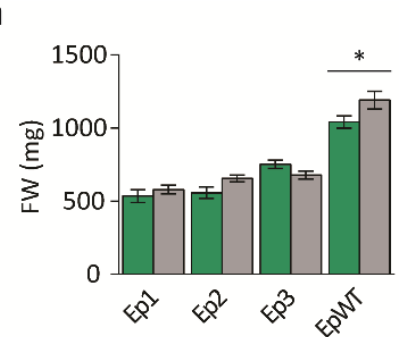

b
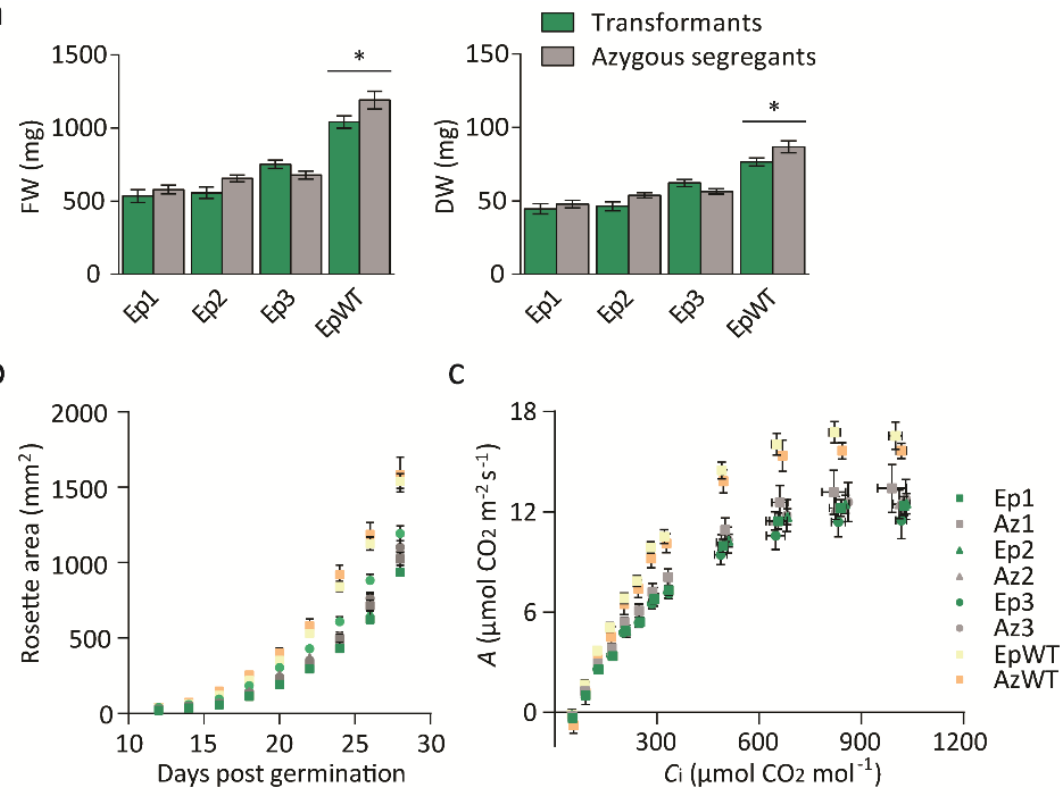

d
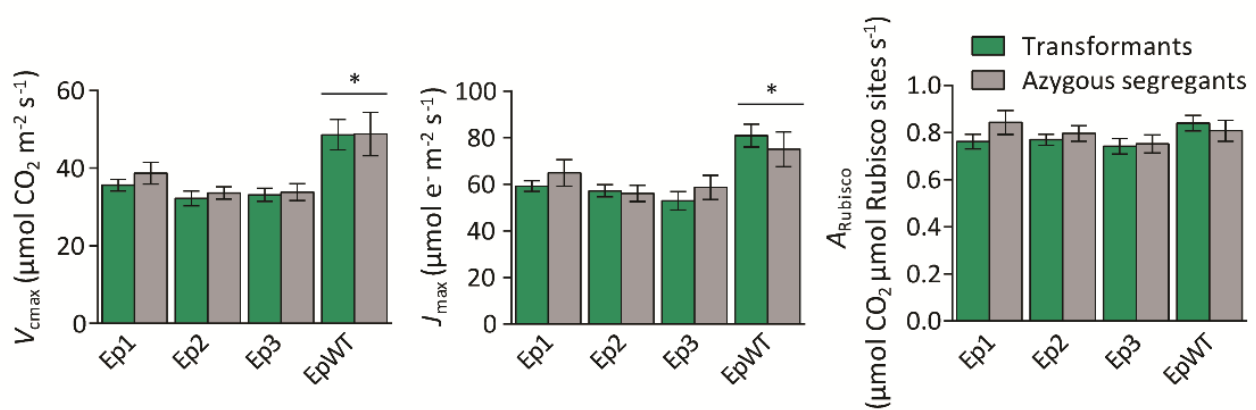

e

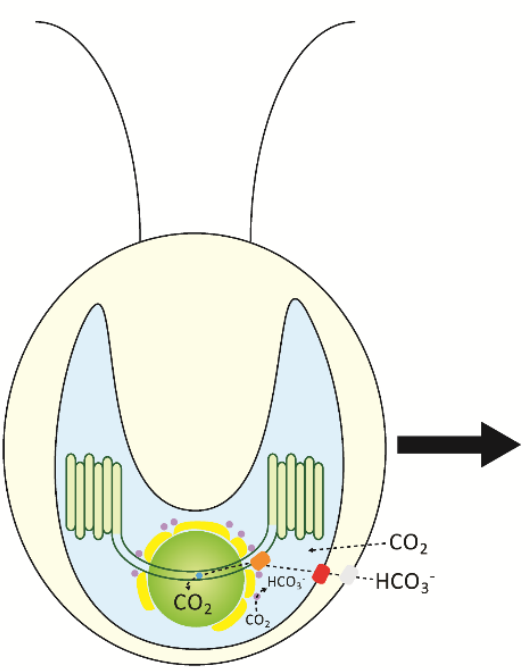

Chlamydomonas
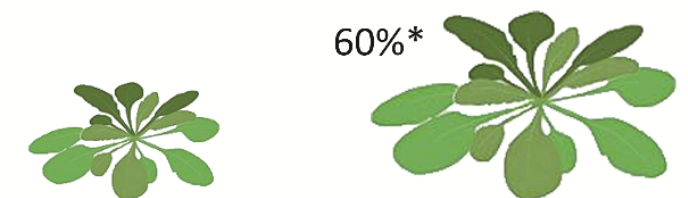

Figure 4. EPYC1-mediated condensation of Rubisco has no negative impact on growth

and photosynthesis. a, Fresh and dry weights of three T2 EPYC1-dGFP S2 $2_{\mathrm{Cr}}$ transgenic lines (Ep1-3) and an EPYC1-dGFP WT transformant (EpWT) (both in green) with their respective azygous segregants (Az1-3 and AzWT) (in grey). Plants were measured after 32 days of growth under $200 \mu \mathrm{mol}$ photons $\mathrm{m}^{-2} \mathrm{~s}^{-1}$ light. The mean and SEM are shown for $\geq 12$ individual plants for each line. b, Rosette expansion of $\mathrm{S} 2 \mathrm{Cr}$ and WT lines in (a). c, Net $\mathrm{CO}_{2}$ assimilation $(A)$ 
564 based on intercellular $\left[\mathrm{CO}_{2}\right]\left(C_{\mathrm{i}}\right)$ under saturating light $\left(1500 \mu \mathrm{mol}\right.$ photons $\left.\mathrm{m}^{-2} \mathrm{~s}^{-1}\right)$. Values

565 show the mean and SEM of measurements made on individual leaves from ten or more

566 individual rosettes. d, Variables derived from gas exchange data include maximum rate of

567 Rubisco carboxylation $\left(V_{\mathrm{cmax}}\right)$, maximum electron transport rate $\left(J_{\max }\right)$, stomatal conductance

$568\left(G_{\mathrm{s}}\right)$, mesophyll conductance $\left(G_{\mathrm{m}}\right)$ and the net $\mathrm{CO}_{2}$ assimilation rate at ambient concentrations

569 of $\mathrm{CO}_{2}$ normalized to Rubisco $\left(A_{\text {Rubisco }}\right)$. Asterisks indicate significant difference $(P<0.05)$ of

570 EpWT lines compared to Ep lines as determined by ANOVA. e, Algal CCM components

571 required for enhancing photosynthesis. Generating a pyrenoid-like condensate in a plant

572 chloroplast provides a platform for introducing bicarbonate $\left(\mathrm{HCO}_{3}{ }^{-}\right)$channels/pumps at the

573 chloroplast envelope (e.g. LCIA, shown in red) ${ }^{33}$ and thylakoid membrane (e.g. BST1-3, shown

574 in orange $)^{35}$, a lumenal carbonic anhydrase to convert $\mathrm{HCO}_{3}{ }^{-}$to $\mathrm{CO}_{2}$ for release into the

575 surrounding Rubisco condensate $(\mathrm{CAH} 3 \text {, shown in blue })^{35}$, mechanisms to capture $\mathrm{CO}_{2}$ as

$576 \mathrm{HCO}_{3}{ }^{-}$(LCIB and LCIC, shown in purple) ${ }^{36,37}$ and traversing thylakoid membranes ${ }^{32}$. Current

577 models suggest that introduction of a functional biophysical CCM into a C3 plant could lead

578 to productivity gains of up to $60 \%{ }^{8,9,29}$. 
Extended Data Table 1. Photosynthetic parameters from gas exchange and fluorescence measurements for $\mathrm{S} 2{ }_{\mathrm{Cr}}$ transgenic lines of Arabidopsis.

580 The mean and SEM are shown for seven 35- to 45 -d-old rosettes for gas exchange variables and twelve 32 -d-old rosettes for $F_{\mathrm{v}} / F_{\mathrm{m}} . F_{\mathrm{v}} / F_{\mathrm{m}}$ is shown for attached leaves dark-adapted for 45 min prior to fluorescence measurements. Letters above the SEM indicate significant difference $(\mathrm{P}<0.05)$ as determined by ANOVA followed by Tukey's HSD tests. Values followed by the same letter are not statistically significantly different. Abbreviations: $\Gamma, \mathrm{CO}_{2}$ compensation point $\left(C_{\mathrm{i}}-A\right) ; F_{\mathrm{v}} / F_{\mathrm{m}}$, maximum potential quantum efficiency of photosystem II; $g_{\mathrm{s}}$, stomatal conductance to water vapour; $g_{\mathrm{m}}$, mesophyll conductance to $\mathrm{CO}_{2}$ (i.e. conductance of $\mathrm{CO}_{2}$ across the pathway from intercellular airspace to chloroplast stroma) using the Ethier and Livingston method ${ }^{48,52} ; J_{\max }$, maximum electron transport rate; $V_{\mathrm{cmax}}$, maximum rate of Rubisco carboxylation.

$V_{\mathrm{cmax}}\left(\mu \mathrm{mol} \mathrm{CO}_{2} \mathrm{~m}^{-2} \mathrm{~s}^{-1}\right)$ $J_{\max }\left(\mu \mathrm{mol} \mathrm{e}^{-} \mathrm{m}^{-2} \mathrm{~s}^{-1}\right)$

$\Gamma\left(\mu \mathrm{mol} \mathrm{CO} \mathrm{CO}^{-2} \mathrm{~s}^{-1}\right)$

$g_{\mathrm{s}}\left(\mathrm{mol} \mathrm{H} \mathrm{H}_{2} \mathrm{~m}^{-2} \mathrm{~s}^{-1}\right)$

$g_{\mathrm{m}}\left(\mathrm{mol} \mathrm{CO} 2 \mathrm{~m}^{-2} \mathrm{~s}^{-1}\right)$

$F_{\mathrm{v}} / F_{\mathrm{m}}(\mathrm{ML})$

$F_{\mathrm{v}} / F_{\mathrm{m}}(\mathrm{HL})$

\begin{tabular}{|c|c|c|c|c|c|c|c|}
\hline Ep1 & $\mathrm{Az} 1$ & Ep2 & $\mathrm{Az2}$ & Ep3 & $\mathrm{Az3}$ & EpWt & $\mathrm{AzWt}$ \\
\hline $35.6 \pm 1.5^{a}$ & $36.4 \pm 2.0^{a}$ & $32.2 \pm 1.9^{a}$ & $33.6 \pm 1.6^{a}$ & $33.1 \pm 1.9^{a}$ & $33.8 \pm 2.2^{a}$ & $44.9 \pm 1.6^{b}$ & $43.3 \pm 1.7^{b}$ \\
\hline $59.2 \pm 2.3^{a}$ & $61.9 \pm 6.3^{a}$ & $57.2 \pm 2.6^{a}$ & $56.1 \pm 3.5^{a}$ & $52.9 \pm 4.4^{a}$ & $58.6 \pm 5.2^{a}$ & $76.4 \pm 2.4^{b}$ & $74.9 \pm 7.5^{b}$ \\
\hline $63 \pm 8^{a}$ & $53 \pm 5^{a}$ & $52 \pm 6^{a}$ & $54 \pm 7^{a}$ & $53 \pm 7^{a}$ & $56 \pm 8^{a}$ & $51 \pm 7^{a}$ & $64 \pm 12^{a}$ \\
\hline $0.249 \pm 0.031^{a}$ & $0.279 \pm 0.051^{a}$ & $0.233 \pm 0.017^{a}$ & $0.251 \pm 0.015^{a}$ & $0.233 \pm 0.021^{a}$ & $0.236 \pm 0.016^{a}$ & $0.287 \pm 0.018^{a}$ & $0.306 \pm 0.011^{a}$ \\
\hline $0.034 \pm 0.001^{b}$ & $0.035 \pm 0.003^{b}$ & $0.032 \pm 0.002^{b}$ & $0.033 \pm 0.002^{b}$ & $0.034 \pm 0.003^{b}$ & $0.032 \pm 0.002^{b}$ & $0.045 \pm 0.002^{a}$ & $0.046 \pm 0.003^{a}$ \\
\hline $0.848 \pm 0.002^{a}$ & $0.849 \pm 0.002^{a}$ & $0.848 \pm 0.001^{a}$ & $0.847 \pm 0.001^{a}$ & $0.847 \pm 0.002^{a}$ & $0.845 \pm 0.002^{a}$ & $0.851 \pm 0.002^{a}$ & $0.850 \pm 0.001^{a}$ \\
\hline $0.852 \pm 0.002^{\mathrm{a}}$ & $0.845 \pm 0.002^{a}$ & $0.850 \pm 0.001^{a}$ & $0.855 \pm 0.004^{a}$ & $0.846 \pm 0.002^{a}$ & $0.849 \pm 0.001^{a}$ & $0.850 \pm 0.003^{a}$ & $0.852 \pm 0.002^{a}$ \\
\hline
\end{tabular}


bioRxiv preprint doi: https://doi.org/10.1101/2020.10.26.354332; this version posted October 27, 2020. The copyright holder for this preprint (which was not certified by peer review) is the author/funder, who has granted bioRxiv a license to display the preprint in perpetuity. It is made available under aCC-BY-NC-ND 4.0 International license.

601

602

603

604

605

606

607

608

609

610

611

612

613

614

615

616

617

618

619

620

621

622

623

624

625

626

627

628

629

630

631

632

633

634

635

636

637

638

639

640

641

642

643

644

645

646

647

Extended Data Figure 1. Full sequence of the EPYC1 expression cassettes. Sequence parts are colour coded, and MoClo overhangs are shown in lower case.
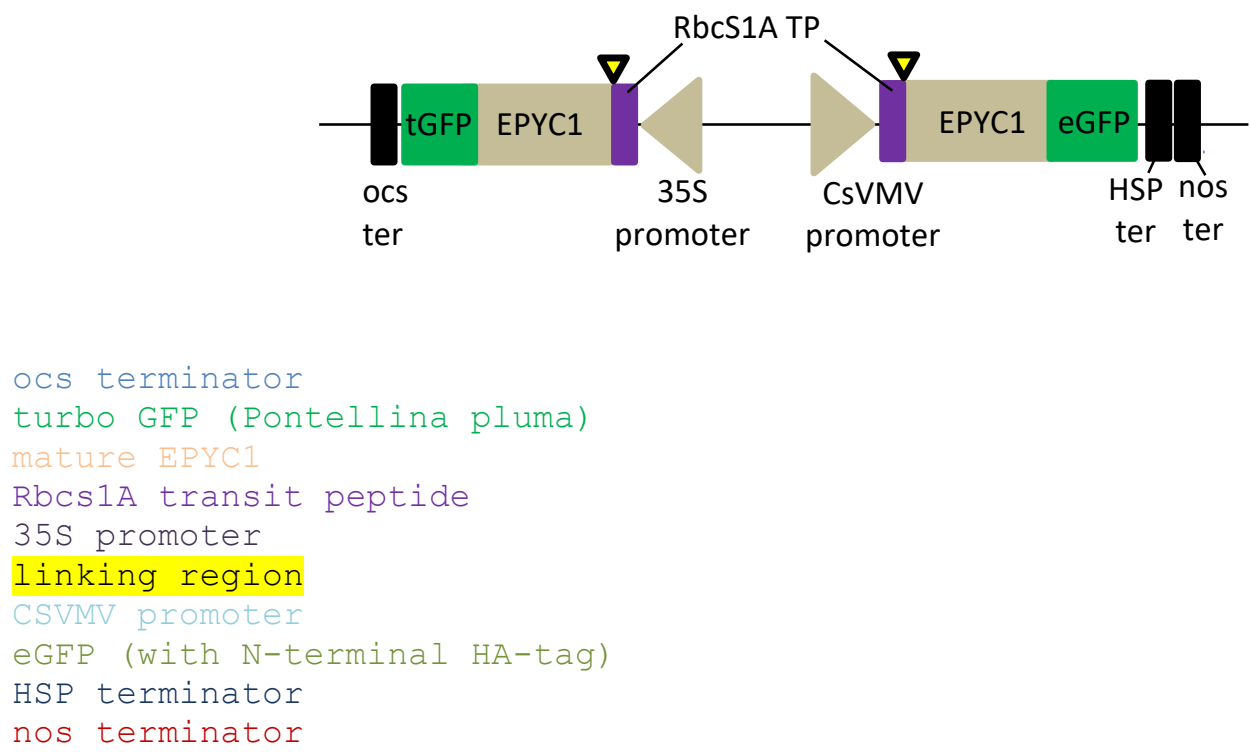

GTCCTGCTGAGCCTCGACATGTTGTCGCAAAATTCGCCCTGGACCCGCCCAACGATTTGTCGTCACTGTCAAGGT TTGACCTGCACTTCATTTGGGGCCCACATACACCAAAAAAATGCTGCATAATTCTCGGGGCAGCAAGTCGGTTAC CCGGCCGCCGTGCTGGACCGGGTTGAATGGTGCCCGTAACTTTCGGTAGAGCGGACGGCCAATACTCAACTTCAA GGAATCTCACCCATGCGCGCCGGCGGGGAACCGGAGTTCCCTTCAGTGAGCGTTATTAGTTCGCCGCTCGGTGTG TCGTAGATACTAGCCCCTGGGGCACTTTTGAAATTTGAATAAGATTTATGTAATCAGTCTTTTAGGTTTGACCGG TTCTGCCGCTTTTTTAAAATTGATTTGTAATAATAAAACGCAATTGTTTGTTATTGTGGCGCTCTATCATAGA TGTCGCTATAAACCTATTCAGCACAATATATTGTTTTCATTTTAATATTGTACATATAAGTAGTAGGGTACAATC AGTAAATTGAACGGAGAATATTATTCATAAAAATACGATAGTAACGGGTGATATATTCATTAGAATGAACCGAAA CCGGCGGTAAGGATCTGAGCTACACATGCTCAGGTTTTTTACAACGTGCACAACAGAATTGAAAGCAAATATCAT GCGATCATAGGCTTCTCGCATATCTCATTAAAGCAGGACaagCTTATCATTCCTCACCAGCATCAGCATCAGGGG TCTTGAAAGCATGTTGGTACTCAACGATTCCAAGCTCGGTGTTAGAGTGATCTTCCTCAACTCTTCTGAAAGCGA ACATAGGTCCACCGTTTTGAAGGATAGAAGGGTGGATAGCAGACTTGAAGTGCATGTGAGAATCCACCACAGAAG AGTAGTAACCACCATCTCTAAGTGAGAAGGTTCTGGTGAAAGATCCATCGAGATCGTTATCTCCCATAGGATGAA GATGCTCAACAGTAGCGTTAGACCTGATGATCTTGTCGGTGAAGATAACAGAATCCTCAGGGAATCCAGTTCCCA TAACCTGCACATCAACAAATTTGGTCATATATTAGAAAAGTTATAAATTAAAATATACACACTTATAAACTACA GAAAAGCAATTGCTATATACTACATTCTTTTATTTTGAAAAAAATATTTGAAATATTATATTACTACTAATTAAT GATAATTATTATATATATATCAAAGGTAGAAGCAGAAACTTACCTTGAAATCTCCGATCACTCTTCCAGCCTCGT ATCTGTAAGAGAAGCTAACGTGAAGAACACCACCATCCTCGTACTTCTCGATCCTAGTGTTGGTGTATCCACCGT TGTTGATAGCATGAAGGAAAGGGTTCTCGTATCCAGATGGGTAAGTTCCGAAGTGGTAGAATCCGTATCCCATAA CGTGAGAAAGAAGGTATGGAGAGAAGGTAAGAGCACCCTTGGTAGACTTCATCTTGTTAGTCATTCTTCCCTGCT CAGGAGTTCCCTCACCACCTCCAACAAGCTCGAACTCAACACCGTTAAGGGTTCCAGTGATTCTACACTCGATTT CCATAGCAGGAAGTCCAGACTCATCAGACTCAGATCCAGATCCTCTcga

acctCCGCAGTTAACTCTTCCGCCGTTGCTTGTGATGGAAGTAATGTCGTTGT TAGCCTTGCGGGTGGCTGGGAAGGCAGCGGAGGACTTAAGTCCGTTGAAAGGAGCGACCATAGTGGCCTGAGCCG GAGAGGCAACCATAGTAGCGGAAGAGAGCATAGAGGAAGCcattGTATCGATAATTGTAAATGTAATTGTAATGT 
bioRxiv preprint doi: https://doi.org/10.1101/2020.10.26.354332; this version posted October 27, 2020. The copyright holder for this preprint (which was not certified by peer review) is the author/funder, who has granted bioRxiv a license to display the preprint in perpetuity. It is made available under aCC-BY-NC-ND 4.0 International license.

648

649

650

651

652

653

654

655

656

657

658

659

660

661

662

663

664

665

666

667

668

669

670

671

672

673

674

675

676

677

678

679

680

681

682

683

684

685

686

687

688

689

690

691

692

693

694
TGTTTGTTGTTTGTTGTGTTGGTAATTGTTGAAAAATGAGCTCTTATACTCGAGCGTGTCCTCTCCAAATGAA ATGAACTTCCTTATATAGAGGAAGGGTCTTGCGAAGGATAGTGGGATTGTGCGTCATCCCTTACGTCAGTGGAGA TGTCACATCAATCCACTTGCTTTGTAGACGTGGTTGGAACCTCTTCTTTTTCCACGATGCTCCTCGTGGGTGGGG GTCCATCTTTGGGACCACTGTCGGCAGAGAGATCTTGAATGATAGCCTTTCCTTTATCGCAATGATGGCATTTGT AGGAGCCACCTTCCTTTTCTACTGTCCTTTCGATGAAGTGACAGATAGCTGGGCAATGGAATCCGAGGAGGTTTC CCGAAATTATCCTTTGTTGAAAAGTCTCAATAGCCCTTTGATCTTCTGAGACTGTATCTTTGACATTTTTGGAGT AGACCAGAGTGTCGTGCTCCACCATGTTGACCCtCGCAAGAATTCAAGCTTggagCCAGAAGGTAATTATCCAAG ATGTAGCATCAAGAATCCAATGTTACGGGAAAAACTATGGAAGTATTATGTAAGCTCAGCAAGAAGCAGATCAA TATGCGGCACATATGCAACCTATGTTCAAAAATGAAGAATGTACAGATACAAGATCCTATACTGCCAGAATACGA AGAAGAATACGTAGAAATTGAAAAAGAAGAACCAGGCGAAGAAAAGAATCTTGATGACGTAAGCACTGACGACAA CAATGAAAAGAAGAAGATAAGGTCGGTGATTGTGAAAGAGACATAGAGGACACATGTAAGGTGGAAAATGTAAGG GCGGAAAGTAACCTTATCACAAAGGAATCTTATCCCCCACTACTTATCCTTTTATATTTTTCCGTGTCATTTTTG CCCTTGAGTTTCCTATATAAGGAACCAAGTTCGGCATTTGTGAAAACAAGAAAAAATTTGGTGTAAGCTATTTT CTTTGAAGTACTGAGGATACAACTTCAGAGAAATTTGTAAGTTTGTaatgGCTTCCTCTATGCTCTCTTCCGCTA CTATGGTTGCCTCTCCGGCTCAGGCCACTATGGTCGCTCCTTTCAACGGACTTAAGTCCTCCGCTGCCTTCCCAG CCACCCGCAAGGCTAACAACGACATTACTTCCATCACAAGCAACGGCGGAAGAGTTAACTGCGGaggt

tcgTACCCATACGATGTTCCTGACTATGCGGGCT ATCCCTATGACGTCCCGGACTATGCAGGATTGTATCCATATGACGTTCCAGATTACGCCACTAGAGCTGCTTACC CATACGATGTTCCTGACTATGCGGGCTATCCCTATGACGTCCCGGACTATGCAGGATTGTATCCATATGACGTTC CAGATTACGCCGTGAGCAAGGGCGAGGAGCTGTTCACCGGGGTGGTGCCCATCCTGGTCGAGCTGGACGGCGACG TAAACGGCCACAAGTTCAGCGTGTCCGGCGAGGGCGAGGGCGATGCCACCTACGGCAAGCTGACCCTGAAGTTCA TCTGCACCACCGGCAAGCTGCCCGTGCCCTGGCCCACCCTCGTGACCACCCTGACCTACGGCGTGCAGTGCTTCA GCCGCTACCCCGACCACATGAAGCAGCACGACTTCTTCAAGTCCGCCATGCCCGAAGGCTACGTCCAGGAGCGCA CCATCTTCTTCAAGGACGACGGCAACTACAAGACCCGCGCCGAGGTGAAGTTCGAGGGCGACACCCTGGTGAACC GCATCGAGCTGAAGGGCATCGACTTCAAGGAGGACGGCAACATCCTGGGGCACAAGCTGGAGTACAACTACAACA GCCACAACGTCTATATCATGGCCGACAAGCAGAAGAACGGCATCAAGGTGAACTTCAAGATCCGCCACAACATCG AGGACGGCAGCGTGCAGCTCGCCGACCACTACCAGCAGAACACCCCCATCGGCGACGGCCCCGTGCTGCTGCCCG ACAACCACTACCTGAGCACCCAGTCCGCCCTGAGCAAAGACCCCAACGAGAAGCGCGATCACATGGTCCTGCTGG AGTTCGTGACCGCCGCCGGGATCACTCTCGGCATGGACGAGCTGTACAAGTAAgcttATATGAAGATGAAGATGA AATATTTGGTGTGTCAAATAAAAAGCTTGTGTGCTTAAGTTTGTGTTTTTTTCTTGGCTTGTTGTGTTATGAATT TGTGGCTTTTTCTAATATTAAATGAATGTAAGATCTCATTATAATGAATAAACAAATGTTTCTATAATCCATTGT GAATGTTTTGTTGGATCTCTTCTGCAGCATATAACTACTGTATGTGCTATGGTATGGACTATGGAATATGATTAA AGATAAGagatgTCAAGCAGATCGTTCAAACATTTGGCAATAAAGTTTCTTAAGATTGAATCCTGTTGCCGGTCT TGCGATGATTATCATATAATTTCTGTTGAATTACGTTAAGCATGTAATAATTAACATGTAATGCATGACGTTATT TATGAGATGGGTTTTATGATTAGAGTCCCGCAATTATACATTTAATACGCGATAGAAAACAAAATATAGCGCGC AAACTAGGATAAATTATCGCGCGCGGTGTCATCTATGTTACTAGATCGA 
695 Extended Data Figure 2. Transcript abundances of tGFP and eGFP in EPYC1-dGFP

696 plant lines. qRT-PCR was carried out on three T2 S2 Cr transgenic plants expressing EPYC1-

697 dGFP (Ep1- Ep3, as in Fig. 1B) using gene-specific primers for tGFP and eGFP. Abundances

698 of tGFP and eGFP transcripts are shown relative to the highest tGFP expression level and 699 normalised to reference genes PP2A (At1g13320) and RHIP1 (At4g26410). Error bars show 700 the SEM for two biological replicates.

701

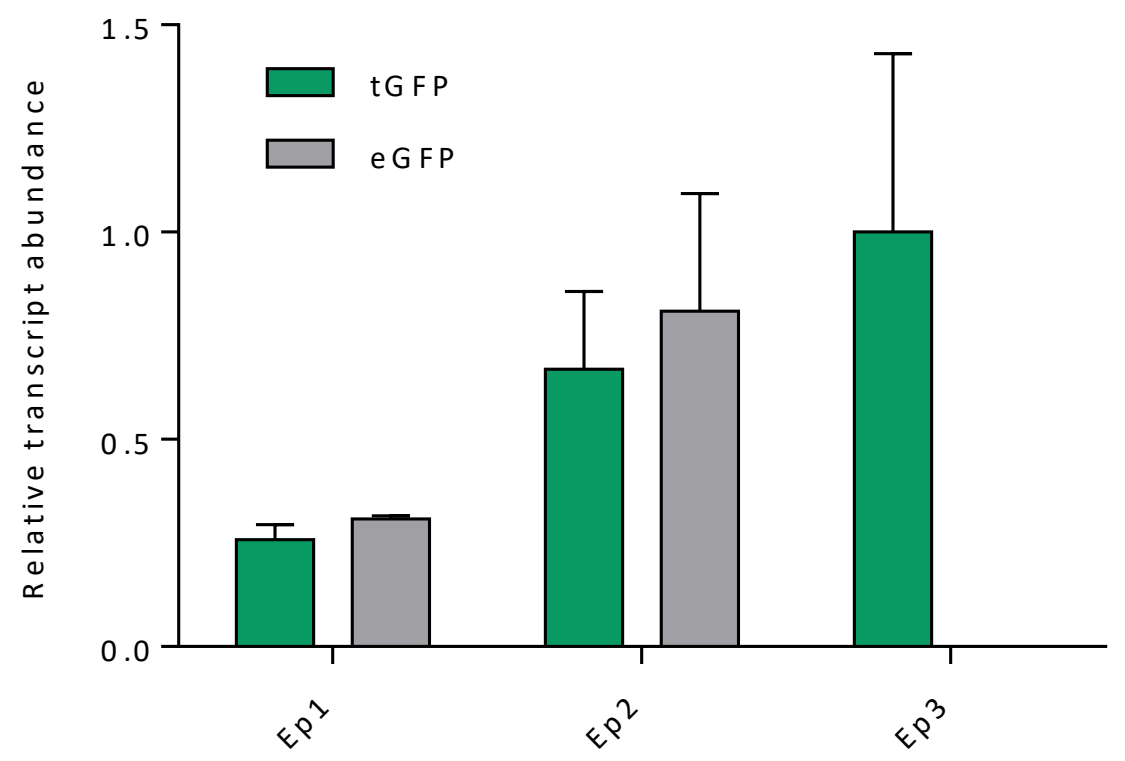


703 Extended Data Figure 3. Characterisation of condensates in S2 Cr plants. a, Maximum

704 projection of a $\mathrm{z}$ stack showing condensates in every chloroplast. Scale bar $=5 \mu \mathrm{m}$. $\mathbf{b}$, The size

705 of condensates is dependent on the expression level of EPYC1. Arabidopsis lines Ep1-3 with

706 different expression levels of EPYC1-dGFP (see Figure. 1b) have different sizes of

707 condensates. Scale bar $=10 \mu \mathrm{m}$. c, Data derived from confocal images of Chlamydomonas

708 pyrenoids $(n=55)$ and chloroplasts $(n=42)$ from each of the three Ep transgenic lines $(E p 1-3)$

709 showing the mean diameter and SEM of pyrenoids and condensates (left). The volume of the

710 condensate is shown plotted against estimated chloroplast volume (centre) and the estimated

711 proportion of chloroplast volume occupied by the condensate (right) ( $n=27$ chloroplasts for

712 each line).

713
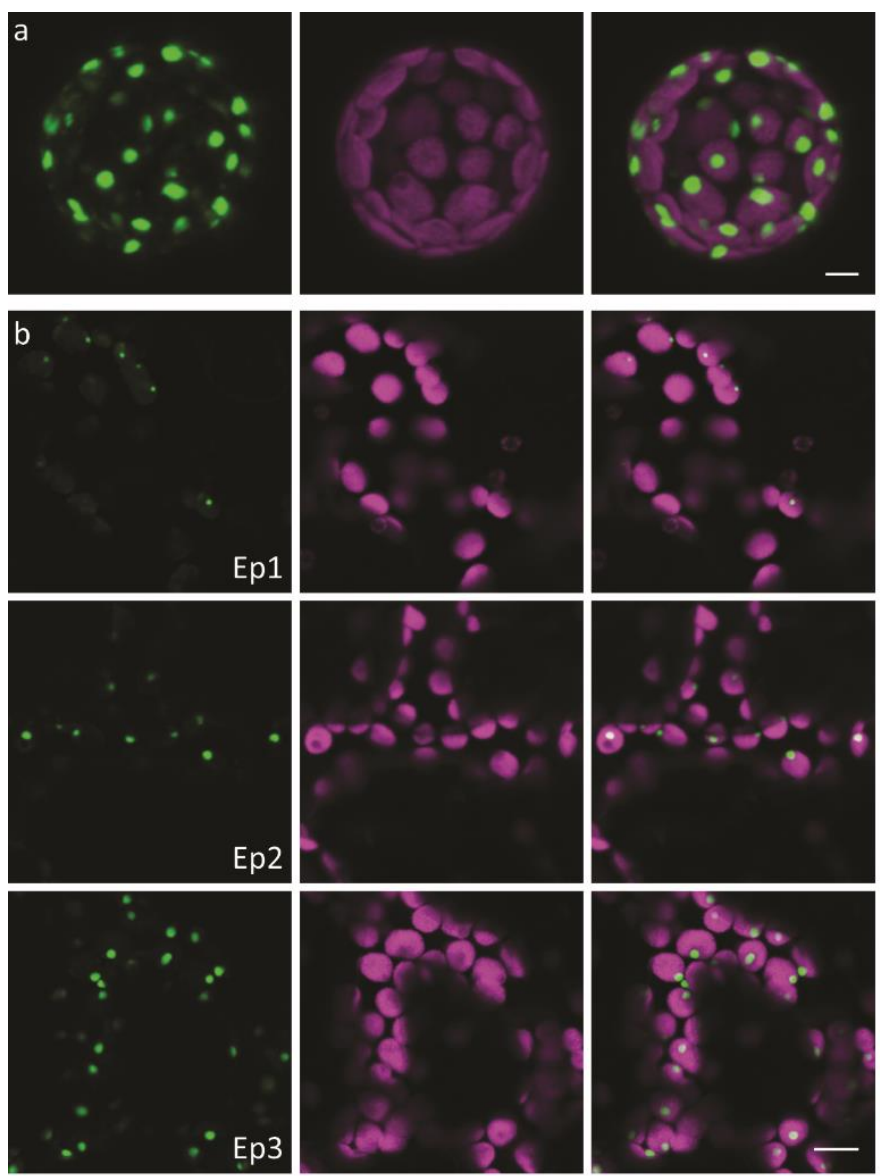

C
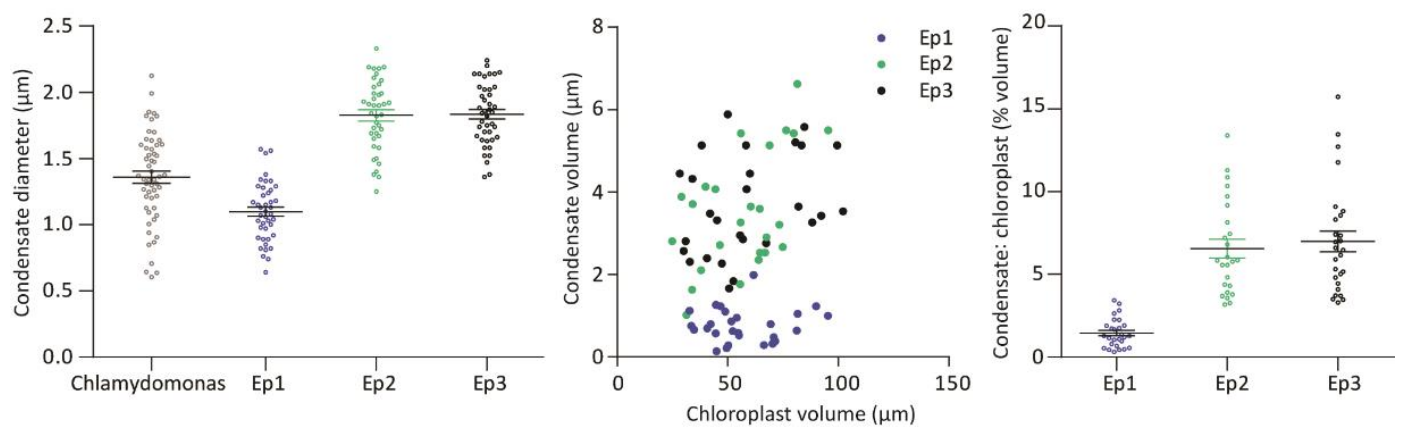
715 Extended Data Figure 4. Condensate formation in $\mathbf{S 2} \mathbf{C r}$ plants. a, Condensation occurs in

716 S2 Cr plants expressing a single EPYC1 expression cassette. Representative images are shown

717 for EPYC1 fused at the C-terminus to either tGFP (top) or eGFP (bottom). Scale bar $=10 \mu \mathrm{m}$.

718 b, Representative TEM image of a mesophyll cell cross-section showing chloroplasts with

719 EPYC1-dGFP condensates. Visible condensates are marked by a white arrowhead. The section

720 has been probed by immunogold labelling with anti-Rubisco antibodies. Scale bar $=1 \mu \mathrm{m}$. c,

721 Representative TEM image of a chloroplast from a wild-type plant expressing EPYC1-dGFP.

722 No condensates were observed in this background. Scale bar $=0.5 \mu \mathrm{m}$.
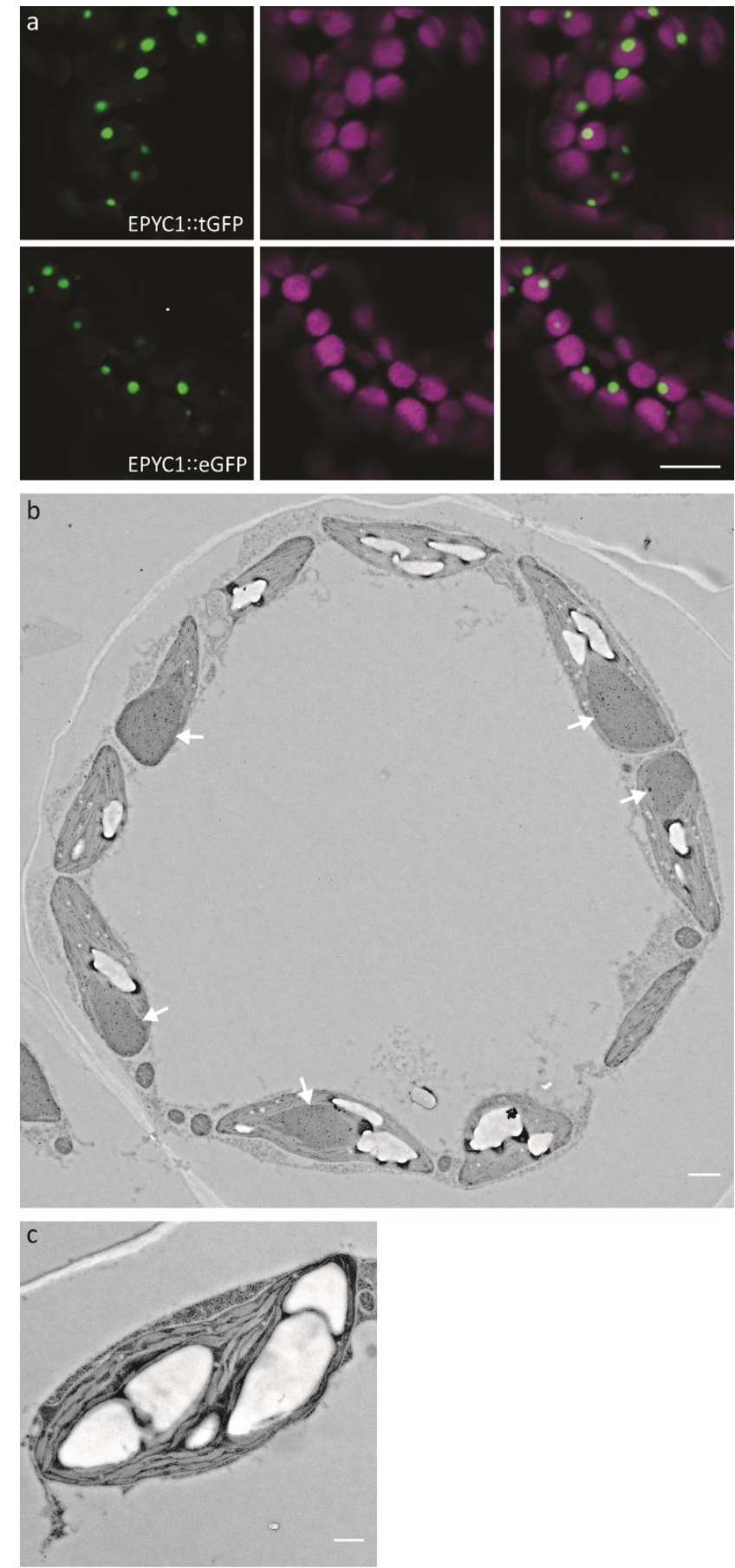


\section{Extended Data Figure 5. Growth of plant lines expressing EPYC1 under different light}

726 levels. a, Fresh (FW) and dry weight (DW) of three T2 EPYC1-dGFP S2 ${ }_{\mathrm{Cr}}$ transgenic lines

727 (Ep1-3) and an EPYC1-dGFP WT transformant (EpWT) with their respective azygous

728 segregants (Az1-3 and AzWT) measured after 32 days of growth at 200 or $900 \mu$ mol photons

$729 \mathrm{~m}^{-2} \mathrm{~s}^{-1}$ light. Asterisks indicate significant difference $(P<0.05)$ as determined by ANOVA. b,

730 Rosette area expansion rates for individual transgenic lines and azygous segregants at two

731 different light levels. The mean and SEM are shown for $\geq 12$ individual plants for each line.

732

a

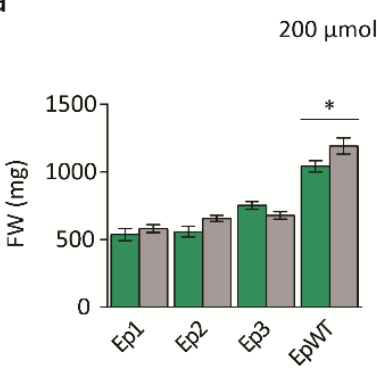

b
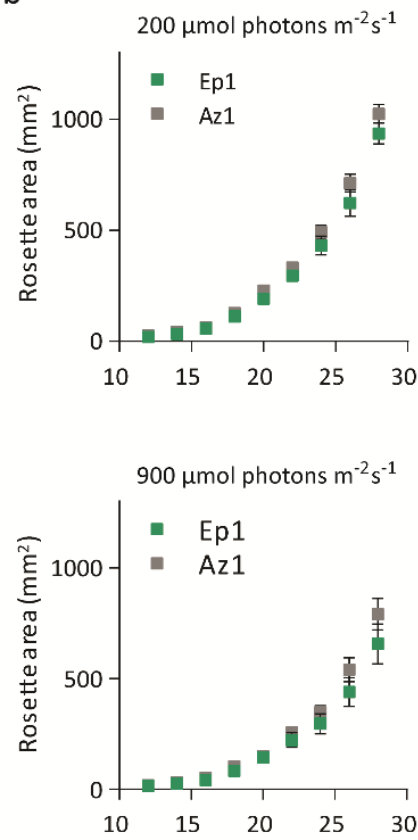
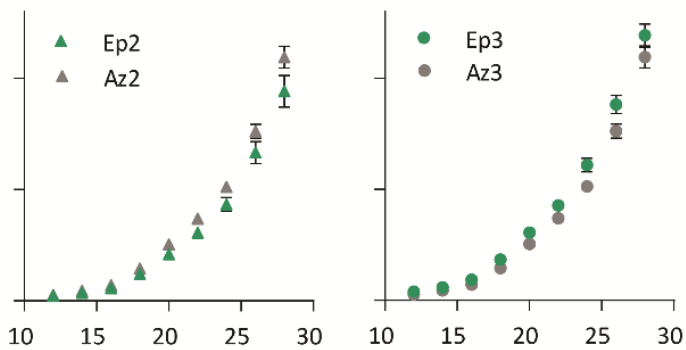

Days post germination
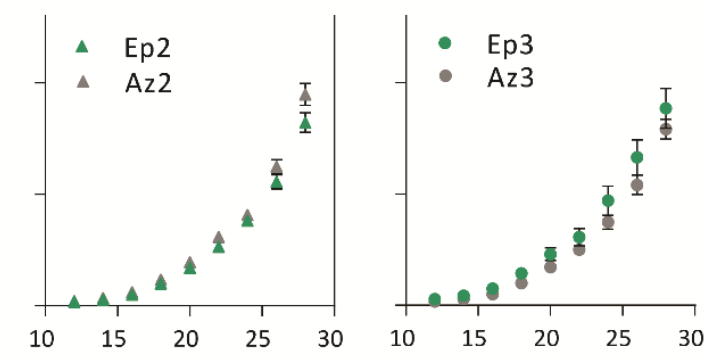

$900 \mu \mathrm{mol}$ photons $\mathrm{m}^{-2} \mathrm{~s}^{-1}$
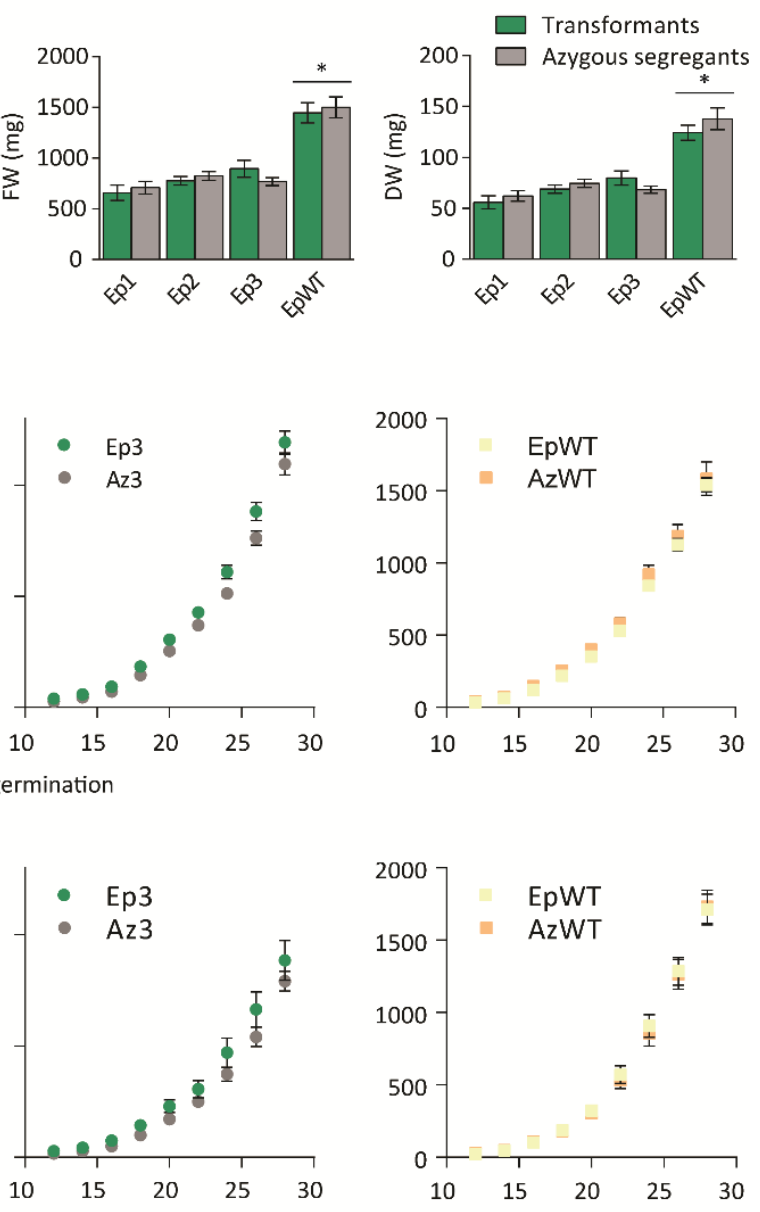